\title{
Dynamics of a delayed SIR model for the transmission of PRRSV among a swine population
}

\author{
Junchen Zou' ${ }^{1}$, Ranjit Kumar Upadhyay², A. Pratap ${ }^{3}$ and Zizhen Zhang ${ }^{1 *}$
}

\author{
"Correspondence: \\ zzzhaida@163.com \\ 'School of Management Science \\ and Engineering, Anhui University \\ of Finance and Economics, Bengbu, \\ 233030, China \\ Full list of author information is \\ available at the end of the article
}

\begin{abstract}
The objective of this paper is to propose a delayed susceptible-infectious-recovered (SIR) model for the transmission of porcine reproductive respiratory syndrome virus (PRRSV) among a swine population, including the latent period delay of the virus and the time delay due to the period the infectious swines need to recover. By taking different combinations of the two delays as the bifurcation parameter, local stability of the disease-present equilibrium and the existence of Hopf bifurcation are analyzed. Sufficient conditions for global stability of the disease-present equilibrium are derived by constructing a suitable Lyapunov function. Directly afterwards, properties of the Hopf bifurcation such as direction and stability are studied with the aid of the normal form theory and center manifold theorem. Finally, numerical simulations are presented to justify the validity of the derived theoretical results.
\end{abstract}

Keywords: Hopf bifurcation; SIR model; Time delay; PRRSV; Periodic solutions

\section{Introduction}

Porcine reproductive respiratory syndrome, also known as blue ear disease and epidemic abortion respiratory syndrome, is caused by Lelystad virus. It was first reported in the United States in 1987 and isolated by Dutch scientists in 1991. Since then it has spread to most of the European pig industry and has a global trend. Porcine reproductive respiratory syndrome has a major economic impact on the swine industry with cost of about 664 million USD annually in the United States [1-3]. In the same way, porcine reproductive respiratory syndrome has also caused huge economic losses to pig industry in China. For example, the porcine reproductive respiratory syndrome epidemic in 2006 forced Chinese farmers to slaughter millions of pigs, leading to China's highest inflation rate in the past decade $[4,5]$.

To better understand the population dynamics of infectious diseases, mathematical models for infectious disease dynamics have been formulated and studied for a long time. For example, delayed epidemic model [6-10], stochastic epidemic model [11-16], agestructured epidemic model [17-19], and so on. Since porcine reproductive respiratory syndrome is a devastating infectious disease among a swine population, it is reasonable to

(c) The Author(s) 2020. This article is licensed under a Creative Commons Attribution 4.0 International License, which permits use, sharing, adaptation, distribution and reproduction in any medium or format, as long as you give appropriate credit to the original author(s) and the source, provide a link to the Creative Commons licence, and indicate if changes were made. The images or other third party material in this article are included in the article's Creative Commons licence, unless indicated otherwise in a credit line to the material. If material is not included in the article's Creative Commons licence and your intended use is not permitted by statutory regulation or exceeds the permitted use, you will need to obtain permission directly from the copyright holder. To view a copy of this licence, visit http://creativecommons.org/licenses/by/4.0/. 
use mathematical modeling describing the propagation of porcine reproductive respiratory syndrome virus among a swine population. Arruda et al. [20-23] proposed different forms of a stochastic model to investigate transmission dynamics of porcine reproductive respiratory syndrome virus. In [24], Suksamran et al. proposed a structured model for the spread of porcine reproductive respiratory syndrome virus incorporating both time and spatial dimensions as well as the decline of infectiousness with time.

Studies have shown that porcine reproductive respiratory syndrome virus is a doubleedged sword to the immune system of pigs [25]. On the one hand, porcine reproductive respiratory syndrome virus can specifically bind to immune cells, especially macrophages, and once it is widely replicated, the immune system will be inhibited, resulting in immune failure of a variety of infectious diseases vaccines; on the other hand, as virus infection stimulates the immune system of pigs, the body produces immunity, which will protect the body from secondary infection. Accordingly, vaccination is an important method used for controlling the spread of porcine reproductive respiratory syndrome virus. Based on this consideration, Phoo-ngurn et al. [26] proposed the following susceptible-infectiousrecovered (SIR) model for the transmission of porcine reproductive respiratory syndrome virus among a swine population:

$$
\left\{\begin{aligned}
\frac{d S(t)}{d t}= & (1-q) b\left(1-\frac{S(t)+I(t)+R(t)}{K}\right)(S(t)+r I(t)+R(t)) \\
& -\beta S(t) I(t)-(\mu+v) S(t), \\
\frac{d I(t)}{d t}= & \beta S(t) I(t)-(\mu+\gamma) I(t) \\
\frac{d R(t)}{d t}= & q b\left(1-\frac{S(t)+I(t)+R(t)}{K}\right)(S(t)+r I(t)+R(t)) \\
& +\gamma I(t)+v S(t)-\mu R(t),
\end{aligned}\right.
$$

where $S(t), I(t)$, and $R(t)$ stand for the numbers of susceptible swines, infectious swines, and the recovered swines at time $t$, respectively. $b$ is the birth rate of the susceptible swines; $q$ denotes vaccination coverage; $K$ is the swine carrying capacity; $\mu$ is the natural death rate of the swines; $\gamma$ is the recovery rate of the infectious swines; $v$ is the vaccination rate of the susceptible swines; $\beta$ is the transmission coefficient and $r$ is the abortion proportion.

One of the significant features of porcine reproductive respiratory syndrome virus is its latent characteristic, which means that when viruses enter a pig, they hide themselves and only become active after a certain period. It is therefore easy to show that when the susceptible swines are infected by porcine reproductive respiratory syndrome virus, there will be a time delay before these swines develop themselves into the infectious ones. Likewise, a certain period of time is usually needed for the infectious swines to recover. On the other hand, many research works about a delayed dynamical system in epidemics [6, 9, 27-29], population dynamics [30-35], and neural networks [36-40] have shown that delay differential equations exhibit much more complicated dynamics than ordinary differential equations since a time delay could cause the equilibrium of a dynamical model to lose its stability and cause the occurrence of Hopf bifurcation, which is not welcome for a dynamical system. Due to these facts and without loss of reality, we consider the following delayed 


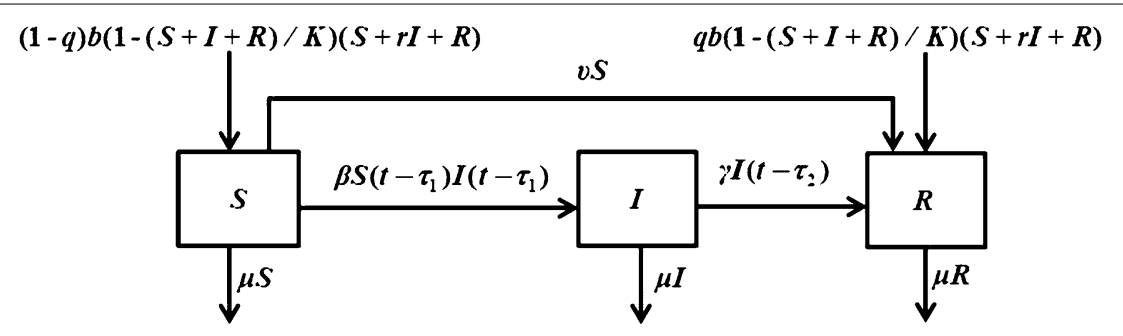

Figure 1 The flow diagram of system (2)

SIR model for the transmission of porcine reproductive respiratory syndrome virus:

$$
\left\{\begin{aligned}
\frac{d S(t)}{d t}= & (1-q) b\left(1-\frac{S(t)+I(t)+R(t)}{K}\right)(S(t)+r I(t)+R(t)) \\
& -\beta S\left(t-\tau_{1}\right) I\left(t-\tau_{1}\right)-(\mu+v) S(t) \\
\frac{d I(t)}{d t}= & \beta S\left(t-\tau_{1}\right) I\left(t-\tau_{1}\right)-\mu I(t)-\gamma I\left(t-\tau_{2}\right) \\
\frac{d R(t)}{d t}= & q b\left(1-\frac{S(t)+I(t)+R(t)}{K}\right)(S(t)+r I(t)+R(t)) \\
& +\gamma I\left(t-\tau_{2}\right)+v S(t)-\mu R(t)
\end{aligned}\right.
$$

where $\tau_{1}$ is latent period delay of the porcine reproductive respiratory syndrome virus; $\tau_{2}$ is time delay due to the period the infectious swines need to recover. The flow diagram for system (2) is illustrated in Fig. 1.

The remainder of this paper is planned as follows. In Sect. 2, sufficient criteria to ensure local stability of the disease-present equilibrium and the existence of Hopf bifurcation of the involved model are presented. In Sect. 3, global stability of the disease-present equilibrium is analyzed. Direction and stability of the Hopf bifurcation are investigated in Sect. 4. Numerical simulations are performed to check our obtained theoretical results in Sect. 5. Finally, a brief conclusion is presented.

\section{Local stability and existence of Hopf bifurcation}

According to the analysis in [26], we know that system (2) has disease-present equilibrium $E^{*}\left(S^{*}, I^{*}, R^{*}\right)$ when the basic reproduction number $R_{0}>1$ where

$$
\begin{aligned}
& S^{*}=\frac{\mu+\gamma}{\beta}, \\
& R^{*}=\frac{(1-q) \mu S^{*}+(1-q) \gamma I^{*}+q \beta S^{*} I^{*}+q(\mu+v) S^{*}}{(1-q) \mu},
\end{aligned}
$$

where $I^{*}$ satisfies

$$
a_{2}\left(I^{*}\right)^{2}+a_{1} I^{*}+a_{0}=0
$$

with

$$
\begin{aligned}
& a_{0}=\left(\frac{b}{\mu}-1\right)\left(\frac{(\mu+v)(\mu+\gamma)}{\beta}\right)-\frac{b(\mu+v)^{2}(\mu+\gamma)^{2}}{K \mu^{2} \beta^{2}(1-q)}, \\
& a_{1}=\left((1-q) b-\frac{b(\mu+v)(\mu+\gamma)}{K \mu \beta}\right)\left(r-1+\frac{\mu+\gamma}{(1-q) \mu}\right)-\frac{b(\mu+v)(\mu+\gamma)^{2}}{K \beta \mu^{2}(1-q)}-(\mu+\gamma),
\end{aligned}
$$




$$
a_{2}=\frac{-b(\mu+\gamma)}{K \mu}\left(r-1+\frac{\mu+\gamma}{(1-q) \mu}\right)
$$

and

$$
R_{0}=(1-q) \frac{\beta(1-\mu / b) \mu K}{(\mu+v)(\mu+\gamma)} .
$$

For the distribution of positive roots of Eq. (3), we have Lemma 1.

Lemma 1 (i) If the coefficients in Eq. (3) satisfy one of the conditions in (a)-(d), then Eq. (3) has one positive root. (a): if $a_{2}=0$ and $a_{0} a_{1}<0$; (b): if $\sqrt{a_{1}^{2}-4 a_{2} a_{0}}>0$ and $a_{0} a_{2}<0$; (c): if $\sqrt{a_{1}^{2}-4 a_{2} a_{0}}>0, a_{0}=0$, and $a_{1} a_{2}<0$; (d): if $\sqrt{a_{1}^{2}-4 a_{2} a_{0}}=0$ and $a_{1} a_{2}<0$; (ii) if the coefficients in Eq. (3) satisfy the condition in (e): $\sqrt{a_{1}^{2}-4 a_{2} a_{0}}>0, a_{1} a_{2}<0$, and $a_{0} a_{2}>0$, then Eq. (3) has two positive roots.

The linear section of system (2) at $E^{*}$ is

$$
\left\{\begin{array}{l}
\frac{d S(t)}{d t}=p_{11} S(t)+p_{12} I(t)+p_{13} R(t)+q_{11} S\left(t-\tau_{1}\right)+q_{12} I\left(t-\tau_{1}\right) \\
\frac{d I(t)}{d t}=p_{22} I(t)+q_{21} S\left(t-\tau_{1}\right)+q_{22} I\left(t-\tau_{1}\right)+s_{22} I\left(t-\tau_{2}\right), \\
\frac{d R(t)}{d t}=p_{31} S(t)+p_{32} I(t)+p_{33} R(t)+s_{32} I\left(t-\tau_{2}\right)
\end{array}\right.
$$

with

$$
\begin{aligned}
& p_{11}=(1-q) b-(\mu+v)-\frac{(1-q) b\left(2 S^{*}+(1+r) I^{*}+2 R^{*}\right)}{K}, \\
& p_{12}=(1-q) b r-\frac{(1-q) b\left((1+r) S^{*}+2 r I^{*}+(1+r) R^{*}\right)}{K}, \\
& p_{13}=(1-q) b-\frac{(1-q) b\left(2 S^{*}+(1+r) I^{*}+2 R^{*}\right)}{K}, \quad p_{22}=-\mu, \\
& p_{31}=q b+v-\frac{q b\left(2 S^{*}+(1+r) I^{*}+2 R^{*}\right)}{K}, \\
& p_{32}=q b r-\frac{q b\left((1+r) S^{*}+2 r I^{*}+(1+r) R^{*}\right)}{K}, \\
& p_{33}=q b-\mu-\frac{q b\left(2 S^{*}+(1+r) I^{*}+2 R^{*}\right)}{K}, \quad q_{21}=\beta I^{*}, \quad q_{22}=\beta S^{*}, \quad s_{22}=-\gamma, \quad s_{32}=\gamma . \\
& q_{11}=-\beta I^{*}, \quad q_{12}=-\beta S^{*}, \quad q, \quad
\end{aligned}
$$

Thus, the associated characteristic equation is

$$
\begin{aligned}
\lambda^{3} & +P_{2} \lambda^{2}+P_{1} \lambda+P_{0}+\left(Q_{2} \lambda^{2}+Q_{1} \lambda+Q_{0}\right) e^{-\lambda \tau_{1}} \\
& +\left(S_{2} \lambda^{2}+S_{1} \lambda+S_{0}\right) e^{-\lambda \tau_{2}}+\left(T_{1} \lambda+T_{0}\right) e^{-\lambda\left(\tau_{1}+\tau_{2}\right)}=0
\end{aligned}
$$

where

$$
\begin{aligned}
& P_{0}=p_{22}\left(p_{13} p_{31}-p_{11} p_{33}\right) \\
& P_{1}=p_{11} p_{22}+p_{11} p_{33}+p_{22} p_{33}-p_{13} p_{31}
\end{aligned}
$$




$$
\begin{aligned}
& P_{2}=-\left(p_{11}+p_{22}+p_{33}\right), \\
& Q_{0}=p_{33}\left(p_{12} q_{21}-p_{22} q_{11}-p_{11} q_{22}\right)-p_{13}\left(p_{31} q_{22}+p_{32} q_{21}\right), \\
& Q_{1}=q_{11}\left(p_{22}+p_{33}\right)+q_{22}\left(p_{11}+p_{33}\right)-p_{12} q_{21}, \\
& Q_{2}=-\left(q_{11}+q_{22}\right), \\
& S_{0}=s_{22}\left(p_{13} p_{31}-p_{11} p_{33}\right), \\
& S_{1}=s_{22}\left(p_{11}+p_{33}\right), \quad S_{2}=-s_{22}, \\
& T_{0}=-\left(p_{13} q_{21} s_{32}+p_{33} q_{11} s_{22}\right), \quad T_{1}=q_{11} s_{22} .
\end{aligned}
$$

Case 1. $\tau_{1}=\tau_{2}=0$. Equation (5) becomes

$$
\lambda^{3}+P_{12} \lambda^{2}+P_{11} \lambda+P_{10}=0
$$

where

$$
P_{10}=P_{0}+Q_{0}+S_{0}+T_{0}, \quad P_{11}=P_{1}+Q_{1}+S_{1}+T_{1}, \quad P_{12}=P_{2}+Q_{2}+S_{2}
$$

Thus, in view of Routh-Hurwitz criteria, if condition $\left(H_{1}\right): P_{10}>0, P_{12}>0$, and $P_{12} P_{11}>$ $P_{10}$ holds, then all roots of Eq. (6) have a negative real part.

Case 2. $\tau_{1}>0, \tau_{2}=0$. Equation (5) becomes

$$
\lambda^{3}+P_{22} \lambda^{2}+P_{21} \lambda+P_{20}+\left(Q_{22} \lambda^{2}+Q_{21} \lambda+Q_{20}\right) e^{-\lambda \tau_{1}}=0
$$

where

$$
\begin{array}{lll}
P_{20}=P_{0}+S_{0}, & P_{21}=P_{1}+S_{1}, & P_{22}=P_{2}+S_{2}, \\
Q_{20}=Q_{0}+T_{0}, & Q_{21}=Q_{1}+T_{1}, & Q_{22}=Q_{2} .
\end{array}
$$

Let $\lambda=i \omega_{1}$ be the root of Eq. (7). Then

$$
\left\{\begin{array}{l}
Q_{21} \omega_{1} \sin \tau_{1} \omega_{1}+\left(Q_{20}-Q_{22} \omega_{1}^{2}\right) \cos \tau_{1} \omega_{1}=P_{22} \omega_{1}^{2}-P_{20} \\
Q_{21} \omega_{1} \cos \tau_{1} \omega_{1}-\left(Q_{20}-Q_{22} \omega_{1}^{2}\right) \sin \tau_{1} \omega_{1}=\omega_{1}^{3}-P_{21} \omega_{1}
\end{array}\right.
$$

Then

$$
\omega_{1}^{6}+U_{22} \omega_{1}^{4}+U_{21} \omega_{1}^{2}+U_{20}=0
$$

with

$$
\begin{aligned}
& U_{20}=P_{20}^{2}-Q_{20}^{2}, \\
& U_{21}=P_{21}^{2}-2 P_{20} P_{22}-Q_{21}^{2}+2 Q_{20} Q_{22}, \\
& U_{22}=P_{22}^{2}-2 P_{21}-Q_{22}^{2} .
\end{aligned}
$$


Let $\varpi_{1}=\omega_{1}^{2}$, then Eq. (9) becomes

$$
\varpi_{1}^{3}+U_{22} \varpi_{1}^{2}+U_{21} \varpi_{1}+U_{20}=0
$$

For the distribution of roots of Eq. (10), we have Lemma 2.

Lemma 2 (i) If $U_{20}<0$, then Eq. (10) has at least one positive root; (ii) If $U_{20} \geq 0$ and $U_{22}^{2}-3 U_{21} \leq 0$, then Eq. (10) has no positive root; (iii) If $U_{20} \geq 0$ and $U_{22}^{2}-3 U_{21}>0$, then Eq. (10) has positive roots if and only if $U_{2 *}=\frac{-U_{22}+\sqrt{U_{22}^{2}-3 U_{21}}}{3}>0$ and $f_{1}\left(U_{2 *}\right) \leq 0$, where $f_{1}\left(\varpi_{1}\right)=\varpi_{1}^{3}+U_{22} \varpi_{1}^{2}+U_{21} \varpi_{1}+U_{20}$.

Assume that $\left(H_{21}\right)$ Eq. (10) has at least one positive root. With loss of generality, we suppose that Eq. (10) has three positive roots denoted by $\varpi_{11}, \varpi_{12}$, and $\varpi_{13}$, respectively. Then Eq. (9) has positive roots $\omega_{1 i}=\sqrt{\varpi_{1 i}}, i=1,2,3$. For $\omega_{1 i}$,

$$
\begin{aligned}
\tau_{1 i}^{(j)}= & \frac{1}{\omega_{1 i}}\left\{\arccos \left[\frac{\left(Q_{21}-P_{22} Q_{22}\right) \omega_{1 i}^{4}+\left(P_{20} Q_{22}-P_{21} Q_{21}+P_{22} Q_{20}\right) \omega_{1 i}^{2}-P_{20} Q_{20}}{Q_{22}^{2} \omega_{1 i}^{4}+\left(Q_{21}^{2}-2 Q_{20} Q_{22}\right) \omega_{1 i}^{2}+Q_{20}^{2}}\right]\right. \\
& +2 j \pi\},
\end{aligned}
$$

where $i=1,2,3$ and $j=0,1,2, \ldots$. Define

$$
\tau_{10}=\tau_{1 i 0}^{(0)}=\min \left\{\tau_{1 i}^{(0)}\right\}, \quad \omega_{10}=\omega_{1 i 0}, \quad i=1,2,3 .
$$

It follows from Eq. (7) that

$$
\left[\frac{d \lambda\left(\tau_{1}\right)}{d \tau_{1}}\right]^{-1}=-\frac{3 \lambda^{2}+2 P_{22} \lambda+P_{21}}{\lambda\left(\lambda^{3}+P_{22} \lambda^{2}+P_{21} \lambda+P_{20}\right)}+\frac{2 Q_{22} \lambda+Q_{21}}{\lambda\left(Q_{22} \lambda^{2}+Q_{21} \lambda+Q_{20}\right)}-\frac{\tau_{1}}{\lambda} .
$$

Further,

$$
\operatorname{Re}\left[\frac{d \lambda\left(\tau_{1}\right)}{d \tau_{1}}\right]_{\tau_{1}=\tau_{10}}^{-1}=\frac{f_{1}^{\prime}\left(\varpi_{10}\right)}{Q_{22}^{2} \omega_{10}^{4}+\left(Q_{21}^{2}-2 Q_{20} Q_{22}\right) \omega_{10}^{2}+Q_{20}^{2}}
$$

where $f_{1}\left(\varpi_{1}\right)=\varpi_{1}^{3}+U_{22} \varpi_{1}^{2}+U_{21} \varpi_{1}+U_{20}$ and $\varpi_{10}=\omega_{10}^{2}$. Then, if $\left(H_{22}\right): f_{1}^{\prime}\left(\varpi_{10}\right) \neq 0$ holds,

$$
\operatorname{sign}\left\{\frac{d(\operatorname{Re}(\lambda))}{d \tau_{1}}\right\}_{\tau_{1}=\tau_{10}}=\operatorname{sign}\left\{\operatorname{Re}\left(\frac{d \lambda}{d \tau_{1}}\right)\right\}_{\tau_{1}=\tau_{10}} \neq 0
$$

Theorem 1 If $R_{0}>1$ and conditions $\left(H_{1}\right),\left(H_{21}\right)$, and $\left(H_{22}\right)$ hold, then the disease-present equilibrium $E^{*}\left(S^{*}, I^{*}, R^{*}\right)$ of system (2) is locally asymptotically stable when $\tau_{1} \in\left[0, \tau_{10}\right)$; system (2) undergoes a Hopf bifurcation when $\tau_{1}=\tau_{10}$ and a family of periodic solutions bifurcate from the disease-present equilibrium $E^{*}\left(S^{*}, I^{*}, R^{*}\right)$ near $\tau_{1}=\tau_{10}$.

Case 3. $\tau_{1}=0, \tau_{2}>0$. Equation (5) becomes

$$
\lambda^{3}+P_{32} \lambda^{2}+P_{31} \lambda+P_{30}+\left(S_{32} \lambda^{2}+S_{31} \lambda+S_{30}\right) e^{-\lambda \tau_{2}}=0
$$


where

$$
\begin{aligned}
& P_{30}=P_{0}+Q_{0}, \quad P_{31}=P_{1}+Q_{1}, \quad P_{32}=P_{2}+Q_{2}, \\
& S_{30}=S_{0}+T_{0}, \quad S_{31}=S_{1}+T_{1}, \quad S_{32}=S_{2} .
\end{aligned}
$$

Let $\lambda=i \omega_{2}$ be the root of Eq. (11). Then

$$
\left\{\begin{array}{l}
S_{31} \omega_{2} \sin \tau_{2} \omega_{2}+\left(S_{30}-S_{32} \omega_{2}^{2}\right) \cos \tau_{2} \omega_{2}=P_{32} \omega_{2}^{2}-P_{30} \\
S_{31} \omega_{2} \cos \tau_{2} \omega_{2}-\left(S_{30}-S_{32} \omega_{2}^{2}\right) \sin \tau_{2} \omega_{2}=\omega_{2}^{3}-P_{31} \omega_{2} .
\end{array}\right.
$$

Then

$$
\omega_{2}^{6}+U_{32} \omega_{2}^{4}+U_{31} \omega_{2}^{2}+U_{30}=0
$$

where

$$
\begin{aligned}
& U_{30}=P_{30}^{2}-S_{30}^{2}, \\
& U_{31}=P_{31}^{2}-2 P_{30} P_{32}-S_{31}^{2}+2 S_{30} S_{32}, \\
& U_{32}=P_{32}^{2}-2 P_{31}-S_{32}^{2} .
\end{aligned}
$$

Let $\varpi_{2}=\omega_{2}^{2}$, then Eq. (13) becomes

$$
\varpi_{2}^{3}+U_{32} \varpi_{2}^{2}+U_{31} \varpi_{2}+U_{30}=0
$$

For the distribution of roots of Eq. (14), we have Lemma 3.

Lemma 3 (i) If $U_{30}<0$, then Eq. (14) has at least one positive root; (ii) If $U_{30} \geq 0$ and $U_{32}^{2}-3 U_{31} \leq 0$, then $E q$. (14) has no positive root; (iii) If $U_{30} \geq 0$ and $U_{32}^{2}-3 U_{31}>0$, then Eq. (10) has positive roots if and only if $U_{3 *}=\frac{-U_{32}+\sqrt{U_{32}^{2}-3 U_{31}}}{3}>0$ and $f_{2}\left(U_{3 *}\right) \leq 0$, where $f_{2}\left(\varpi_{2}\right)=\varpi_{2}^{3}+U_{32} \varpi_{2}^{2}+U_{31} \varpi_{2}+U_{30}$.

Similar to Case 2, assume that $\left(H_{31}\right)$ Eq. (14) has three positive roots denoted by $\varpi_{21}$, $\varpi_{22}$, and $\varpi_{23}$, respectively. Then Eq. (13) has positive roots $\omega_{2 i}=\sqrt{\varpi_{2 i}}, i=1,2,3$. For $\omega_{2 i}$,

$$
\tau_{2 i}^{(j)}=\frac{1}{\omega_{2 i}}\left\{\arccos \left[\frac{\left(S_{31}-P_{32} S_{32}\right) \omega_{2 i}^{4}+\left(P_{30} S_{32}-P_{31} S_{31}+P_{32} S_{30}\right) \omega_{2 i}^{2}-P_{30} S_{30}}{S_{32}^{2} \omega_{2 i}^{4}+\left(S_{31}^{2}-2 S_{30} S_{32}\right) \omega_{2 i}^{2}+S_{30}^{2}}\right]+2 j \pi\right\},
$$

where $i=1,2,3$ and $j=0,1,2, \ldots$. Define

$$
\tau_{20}=\tau_{2 i 0}^{(0)}=\min \left\{\tau_{2 i}^{(0)}\right\}, \quad \omega_{20}=\omega_{2 i 0}, \quad i=1,2,3 .
$$

Then

$$
\left[\frac{d \lambda\left(\tau_{2}\right)}{d \tau_{2}}\right]^{-1}=-\frac{3 \lambda^{2}+2 P_{32} \lambda+P_{31}}{\lambda\left(\lambda^{3}+P_{32} \lambda^{2}+P_{31} \lambda+P_{30}\right)}+\frac{2 S_{32} \lambda+S_{31}}{\lambda\left(S_{32} \lambda^{2}+S_{31} \lambda+S_{30}\right)}-\frac{\tau_{2}}{\lambda} .
$$


Further,

$$
\operatorname{Re}\left[\frac{d \lambda\left(\tau_{2}\right)}{d \tau_{2}}\right]_{\tau_{2}=\tau_{20}}^{-1}=\frac{f_{2}^{\prime}\left(\varpi_{20}\right)}{S_{32}^{2} \omega_{20}^{4}+\left(S_{31}^{2}-2 S_{30} S_{32}\right) \omega_{20}^{2}+Q_{30}^{2}},
$$

where $f_{2}\left(\varpi_{2}\right)=\varpi_{2}^{3}+U_{32} \varpi_{2}^{2}+U_{31} \varpi_{2}+U_{30}$ and $\varpi_{20}=\omega_{20}^{2}$. Then, if $\left(H_{32}\right): f_{2}^{\prime}\left(\varpi_{20}\right) \neq 0$ holds,

$$
\operatorname{sign}\left\{\frac{d(\operatorname{Re}(\lambda))}{d \tau_{2}}\right\}_{\tau_{2}=\tau_{20}}=\operatorname{sign}\left\{\operatorname{Re}\left(\frac{d \lambda}{d \tau_{2}}\right)\right\}_{\tau_{2}=\tau_{20}} \neq 0
$$

Theorem 2 If $R_{0}>1$ and conditions $\left(H_{1}\right),\left(H_{31}\right)$, and $\left(H_{32}\right)$ hold, then the disease-present equilibrium $E^{*}\left(S^{*}, I^{*}, R^{*}\right)$ of system (2) is locally asymptotically stable when $\tau_{2} \in\left[0, \tau_{20}\right)$; system (2) undergoes a Hopf bifurcation when $\tau_{2}=\tau_{20}$ and a family of periodic solutions bifurcate from the disease-present equilibrium $E^{*}\left(S^{*}, I^{*}, R^{*}\right)$ near $\tau_{2}=\tau_{20}$.

Case 4. $\tau_{1}=\tau_{2}=\tau>0$. Equation (5) becomes

$$
\lambda^{3}+P_{42} \lambda^{2}+P_{41} \lambda+P_{40}+\left(Q_{42} \lambda^{2}+Q_{41} \lambda+Q_{40}\right) e^{-\lambda \tau}+\left(T_{41} \lambda+T_{40}\right) e^{-2 \lambda \tau}=0,
$$

where

$$
\begin{aligned}
& P_{40}=P_{0}, \quad P_{41}=P_{1}, \quad P_{42}=P_{2}, \\
& Q_{40}=Q_{0}+S_{0}, \quad Q_{41}=Q_{1}+S_{1}, \quad Q_{42}=Q_{2}+S_{2}, \\
& T_{40}=T_{0}, \quad T_{41}=T_{1} .
\end{aligned}
$$

Multiplying by $e^{\lambda \tau}$, Eq. (15) becomes

$$
Q_{42} \lambda^{2}+Q_{41} \lambda+Q_{40}+\left(\lambda^{3}+P_{42} \lambda^{2}+P_{41} \lambda+P_{40}\right) e^{\lambda \tau}+\left(T_{41} \lambda+T_{40}\right) e^{-\lambda \tau}=0 .
$$

Let $\lambda=i \omega$ be the root of Eq. (16). Then

$$
\left\{\begin{array}{l}
\left(P_{40}-T_{40}-P_{42} \omega^{2}\right) \sin \tau \omega+\left(\left(P_{41}+T_{41}\right) \omega-\omega^{3}\right) \cos \tau \omega=-Q_{41} \omega, \\
\left(P_{40}+T_{40}-P_{42} \omega^{2}\right) \cos \tau \omega-\left(\left(P_{41}-T_{41}\right) \omega-\omega^{3}\right) \sin \tau \omega=Q_{42} \omega^{2}-Q_{40} .
\end{array}\right.
$$

Thus, one can obtain the expressions of $\cos \tau \omega$ and $\sin \tau \omega$ as follows:

$$
\cos \tau \omega=\frac{L_{24} \omega^{4}+L_{22} \omega^{2}+L_{20}}{\omega^{6}+L_{14} \omega^{4}+L_{12} \omega^{2}+L_{10}}, \quad \sin \tau \omega=\frac{L_{25} \omega^{5}+L_{23} \omega^{3}+L_{21} \omega}{\omega^{6}+L_{14} \omega^{4}+L_{12} \omega^{2}+L_{10}},
$$

with

$$
\begin{aligned}
& L_{10}=-T_{40}^{2}, \quad L_{12}=P_{42}^{2}-2 P_{40} P_{42}-T_{41}^{2}, \quad L_{14}=P_{42}^{2}-2 P_{41}, \\
& L_{20}=-\left(P_{40}-Q_{40}\right), \quad L_{21}=\left(P_{41}+T_{41}\right) Q_{40}-\left(P_{40}+T_{40}\right) Q_{41}, \\
& L_{22}=\left(P_{40}-T_{40}\right) Q_{40}-\left(P_{41}-T_{41}\right) Q_{41}+P_{42} Q_{40}, \\
& L_{23}=P_{42} Q_{41}-Q_{40}-\left(P_{41}+T_{41}\right) Q_{42}, \quad L_{24}=Q_{41}-P_{42} Q_{42}, \quad L_{25}=Q_{42} .
\end{aligned}
$$


Further one can obtain the equation with respect to $\omega$ as follows:

$$
\omega^{12}+L_{35} \omega^{10}+L_{34} \omega^{8}+L_{33} \omega^{6}+L_{32} \omega^{4}+L_{31} \omega^{2}+L_{30}=0
$$

with

$$
\begin{aligned}
& L_{30}=L_{10}^{2}-L_{20}^{2}, \quad L_{31}=2 L_{10} L_{12}-2 L_{20} L_{22}-L_{21}^{2}, \\
& L_{32}=L_{12}^{2}+2 L_{10} L_{14}-L_{22}^{2}-2 L_{20} L_{24}-2 L_{21} L_{23}, \\
& L_{33}=2 L_{10}+2 L_{12} L_{14}+2 L_{22} L_{24}-L_{23}^{2}-2 L_{21} L_{25}, \\
& L_{34}=L_{14}^{2}+2 L_{12}-L_{24}^{2}-2 L_{23} L_{25}, \quad L_{35}=2 L_{14}-L_{25}^{2} .
\end{aligned}
$$

Let $\varpi=\omega^{2}$. Then Eq. (18) becomes

$$
\varpi^{6}+L_{35} \varpi^{5}+L_{34} \varpi^{4}+L_{33} \varpi^{3}+L_{32} \varpi^{2}+L_{31} \varpi+L_{30}=0 .
$$

If all the coefficients of system (2) are given, then we can obtain all the coefficients of Eq. (19). Therefore, we assume that $\left(H_{41}\right)$ : Eq. (19) has six positive roots denoted by $\varpi_{1}, \varpi_{2}, \ldots, \varpi_{6}$, respectively. Then, Eq. (18) has positive roots $\omega_{i}=\sqrt{\bar{\varpi}_{i}}, i=1,2, \ldots, 6$. For $\omega_{i}$,

$$
\tau_{i}^{(j)}=\frac{1}{\omega_{i}}\left\{\arccos \left[\frac{L_{24} \omega_{i}^{4}+L_{22} \omega_{i}^{2}+L_{20}}{\omega_{i}^{6}+L_{14} \omega_{i}^{4}+L_{12} \omega_{i}^{2}+L_{10}}\right]+2 j \pi\right\}
$$

where $i=1,2, \ldots, 6$ and $j=0,1,2, \ldots$ Define

$$
\tau_{0}=\tau_{i 0}^{(0)}=\min \left\{\tau_{i}^{(0)}\right\}, \quad \omega_{0}=\omega_{i 0}, \quad i=1,2, \ldots, 6
$$

It follows from Eq. (16) that

$$
\left[\frac{d \lambda(\tau)}{d \tau}\right]^{-1}=-\frac{2 Q_{42} \lambda+Q_{41}+\left(3 \lambda^{2}+2 P_{42} \lambda+P_{41}\right) e^{\lambda \tau}+T_{41} e^{-\lambda \tau}}{\left(\lambda^{4}+P_{42} \lambda^{3}+P_{41} \lambda^{2}+P_{40} \lambda\right) e^{\lambda \tau}-\left(T_{41} \lambda^{2}+T_{40} \lambda\right) e^{-\lambda \tau}}-\frac{\tau}{\lambda},
$$

which leads to

$$
\operatorname{Re}\left[\frac{d \lambda}{d \tau}\right]_{\tau=\tau_{0}}^{-1}=-\frac{L_{41} L_{43}+L_{42} L_{44}}{L_{43}^{2}+L_{44}^{2}}
$$

where

$$
\begin{aligned}
& L_{41}=\left(P_{41}+T_{41}-3 \omega_{0}^{2}\right) \cos \tau_{0} \omega_{0}-2 P_{42} \omega_{0} \cos \tau_{0} \omega_{0}+Q_{41}, \\
& L_{42}=\left(P_{41}-T_{41}-3 \omega_{0}^{2}\right) \sin \tau_{0} \omega_{0}+2 P_{42} \omega_{0} \sin \tau_{0} \omega_{0}+2 Q_{42} \omega_{0}, \\
& L_{43}=\left(\omega_{0}^{4}-P_{41} \omega_{0}^{2}+T_{41} \omega_{0}^{2}\right) \cos \tau_{0} \omega_{0}-\left(P_{40} \omega_{0}+T_{40} \omega_{0}-P_{42} \omega_{0}^{3}\right) \sin \tau_{0} \omega_{0}, \\
& L_{44}=\left(\omega_{0}^{4}-P_{41} \omega_{0}^{2}-T_{41} \omega_{0}^{2}\right) \sin \tau_{0} \omega_{0}+\left(P_{40} \omega_{0}-T_{40} \omega_{0}-P_{42} \omega_{0}^{3}\right) \cos \tau_{0} \omega_{0} .
\end{aligned}
$$


Obviously, if $\left(H_{42}\right): L_{41} L_{43}+L_{42} L_{44} \neq 0$ holds,

$$
\operatorname{sign}\left\{\frac{d(\operatorname{Re}(\lambda))}{d \tau}\right\}_{\tau=\tau_{0}}=\operatorname{sign}\left\{\operatorname{Re}\left(\frac{d \lambda}{d \tau}\right)\right\}_{\tau=\tau_{0}} \neq 0
$$

Theorem 3 If $R_{0}>1$ and conditions $\left(H_{1}\right),\left(H_{41}\right)$, and $\left(H_{42}\right)$ hold, then the disease-present equilibrium $E^{*}\left(S^{*}, I^{*}, R^{*}\right)$ of system (2) is locally asymptotically stable when $\tau \in\left[0, \tau_{0}\right)$; system (2) undergoes a Hopf bifurcation when $\tau=\tau_{0}$ and a family of periodic solutions bifurcate from the disease-present equilibrium $E^{*}\left(S^{*}, I^{*}, R^{*}\right)$ near $\tau=\tau_{0}$.

Case 5. $\tau_{1}>0, \tau_{2} \in\left(0, \tau_{20}\right)$. Let $\lambda=i \omega_{1 *}$ be the root of Eq. (5), then

$$
\left\{\begin{array}{l}
L_{51}\left(\omega_{1 *}\right) \sin \tau_{1} \omega_{1 *}+L_{52}\left(\omega_{1 *}\right) \cos \tau_{1} \omega_{1 *}=L_{53}\left(\omega_{1 *}\right) \\
L_{51}\left(\omega_{1 *}\right) \cos \tau_{1} \omega_{1 *}-L_{52}\left(\omega_{1 *}\right) \sin \tau_{1} \omega_{1 *}=L_{54}\left(\omega_{1 *}\right)
\end{array}\right.
$$

where

$$
\begin{aligned}
& L_{51}\left(\omega_{1 *}\right)=Q_{1} \omega_{1 *}+T_{1} \omega_{1 *} \cos \tau_{2} \omega_{1 *}-T_{0} \sin \tau_{2} \omega_{1 *}, \\
& L_{52}\left(\omega_{1 *}\right)=Q_{0}-Q_{2} \omega_{1 *}^{2}+T_{1} \omega_{1 *} \sin \tau_{2} \omega_{1 *}+T_{0} \cos \tau_{2} \omega_{1 *}, \\
& L_{53}\left(\omega_{1 *}\right)=P_{2} \omega_{1 *}^{2}-P_{0}-S_{1} \omega_{1 *} \sin \tau_{2} \omega_{1 *}-\left(S_{0}-S_{2} \omega_{1 *}^{2}\right) \cos \tau_{2} \omega_{1 *}, \\
& L_{54}\left(\omega_{1 *}\right)=\omega_{1 *}^{3}-P_{1} \omega_{1 *}-S_{1} \omega_{1 *} \cos \tau_{2} \omega_{1 *}+\left(S_{0}-S_{2} \omega_{1 *}^{2}\right) \sin \tau_{2} \omega_{1 *} .
\end{aligned}
$$

Thus, one can obtain the following equation with respect to $\omega_{1 *}$ :

$$
L_{60}\left(\omega_{1 *}\right)+L_{61}\left(\omega_{1 *}\right) \cos \tau_{2} \omega_{1 *}+L_{62}\left(\omega_{1 *}\right) \sin \tau_{2} \omega_{1 *}=0
$$

with

$$
\begin{aligned}
& L_{60}\left(\omega_{1 *}\right)= \omega_{1 *}^{6}+\left(P_{2}^{2}-2 P_{1}-S_{2}^{2}-Q_{2}^{2}\right) \omega_{1 *}^{4} \\
&+\left(P_{1}^{2}-2 P-0 P_{2}+S_{1}^{2}-2 S_{0} S_{2}-Q_{1}^{2}+2 Q_{0} Q_{2}-T_{1}^{2}\right) \omega_{1 *}^{2} \\
&+P_{0}^{2}+S_{0}^{2}-T_{0}^{2}, \\
& L_{61}\left(\omega_{1 *}\right)=2\left[\left(P_{2} S_{2}-S_{1}\right) \omega_{1 *}^{4}+\left(P_{1} S_{1}-P_{0} S_{2}-P_{2} S_{0}-Q_{1} T_{1}+Q_{2} T_{0}\right) \omega_{1 *}^{2}\right. \\
&\left.+P_{0} S_{0}-Q_{0} T_{0}\right], \\
& L_{62}\left(\omega_{1 *}\right)= 2\left[-S_{2} \omega_{1 *}^{5}+\left(P_{1} S_{2}-P_{2} S_{1}+S_{0}+Q_{2} T_{1}\right) \omega_{1 *}^{3}\right. \\
&\left.+\left(P_{0} S_{1}-S_{0} P_{1}-Q_{0} T_{1}+Q_{1} T_{0}\right) \omega_{1 *}\right] .
\end{aligned}
$$

Similar as in Case 4, we suppose that Eq. (22) has at least one positive root. Without loss of generality, we denote the positive roots of Eq. (22) as $\omega_{11}^{*}, \omega_{12}^{*}, \ldots, \omega_{1 k}^{*}$, respectively. For $\omega_{1 i}^{*}, i=0,1, \ldots, k$,

$$
\tau_{1 i}^{*(j)}=\frac{1}{\omega_{1 i}^{*}}\left\{\arccos \left[\frac{l_{51}\left(\omega_{1 i}^{*}\right) \times L_{54}\left(\omega_{1 i}^{*}\right)+L_{52}\left(\omega_{1 i}^{*}\right) \times L_{53}\left(\omega_{1 i}^{*}\right)}{L_{51}^{2}\left(\omega_{1 i}^{*}\right)+L_{52}^{2}\left(\omega_{1 i}^{*}\right)}\right]+2 j \pi\right\},
$$


where $i=1,2, \ldots, k$ and $j=0,1,2, \ldots$ Define

$$
\tau_{10}^{*}=\tau_{1 i 0}^{*(0)}=\min \left\{\tau_{1 i}^{*(0)}\right\}, \quad \omega_{10}^{*}=\omega_{1 i 0}, \quad i=1,2, \ldots, k .
$$

Then

$$
\left[\frac{d \lambda\left(\tau_{1}\right)}{d \tau_{1}}\right]^{-1}=\frac{L_{71}(\lambda)}{L_{72}(\lambda)}-\frac{\tau_{1}}{\lambda}
$$

with

$$
\begin{aligned}
L_{71}(\lambda)= & 3 \lambda^{2}+2 P_{2} \lambda+P_{1}+\left(2 Q_{2} \lambda+Q_{1}\right) e^{-\lambda \tau_{1}} \\
& +\left(-\tau_{2} S_{2} \lambda^{2}+\left(2 S_{2}-\tau_{2} S_{1}\right) \lambda+S_{1}-\tau_{2} S_{0}\right) e^{-\lambda \tau_{2}} \\
& +\left(-\tau_{2} T_{1} \lambda+T_{1}-\tau_{2} T_{0}\right) e^{-\lambda\left(\tau_{1}+\tau_{2}\right)}, \\
L_{72}(\lambda)= & \left(Q_{2} \lambda^{3}+Q_{1} \lambda^{2}+Q_{0} \lambda\right) e^{-\lambda \tau_{1}}+\left(T_{1} \lambda^{2}+T_{0} \lambda\right) e^{-\lambda\left(\tau_{1}+\tau_{2}\right)} .
\end{aligned}
$$

Further,

$$
\operatorname{Re}\left[\frac{d \lambda}{d \tau_{1}}\right]_{\tau_{1}=\tau_{10}^{*}}^{-1}=\frac{L_{81} L_{83}+L_{82} L_{84}}{L_{83}^{2}+L_{84}^{2}}
$$

where

$$
\begin{aligned}
L_{81}= & 3\left(\omega_{10}^{*}\right)^{2}+P_{1}+2 Q_{2} \omega_{10}^{*} \sin \tau_{10}^{*} \omega_{10}^{*}+Q_{1} \cos \tau_{10}^{*} \omega_{10}^{*} \\
& +\left(2 S_{2}-\tau_{2} S_{1}\right) \sin \tau_{2} \omega_{10}^{*}+\left(\tau_{2} S_{2}\left(\omega_{10}^{*}\right)^{2}+S_{1}-\tau_{2} S_{0}\right) \cos \tau_{2} \omega_{10}^{*} \\
& -\tau_{2} T_{2} \omega_{10}^{*} \sin \left(\tau_{10}^{*}+\tau_{2}\right)+\left(T_{1}-\tau_{2} T_{0}\right) \cos \left(\tau_{10}^{*}+\tau_{2}\right), \\
L_{82}= & 2 P_{2} L_{81} \omega_{10}^{*}+2 Q_{2} \omega_{10}^{*} \cos \tau_{10}^{*} \omega_{10}^{*}-Q_{1} \sin \tau_{10}^{*} \omega_{10}^{*} \\
& +\left(2 S_{2}-\tau_{2} S_{1}\right) \cos \tau_{2} \omega_{10}^{*}-\left(\tau_{2} S_{2}\left(\omega_{10}^{*}\right)^{2}+S_{1}-\tau_{2} S_{0}\right) \sin \tau_{2} \omega_{10}^{*} \\
& -\tau_{2} T_{2} \omega_{10}^{*} \cos \left(\tau_{10}^{*}+\tau_{2}\right)-\left(T_{1}-\tau_{2} T_{0}\right) \sin \left(\tau_{10}^{*}+\tau_{2}\right), \\
L_{83}= & \left(Q_{0} \omega_{10}^{*}-Q_{2}\left(\omega_{10}^{*}\right)^{3}\right) \sin \tau_{10}^{*} \omega_{10}^{*}-Q_{1}\left(\omega_{10}^{*}\right)^{2} \cos \tau_{10}^{*} \omega_{10}^{*} \\
& +T_{0} \omega_{10}^{*} \sin \left(\tau_{10}^{*}+\tau_{2}\right)-T_{1}\left(\omega_{10}^{*}\right)^{2} \cos \left(\tau_{10}^{*}+\tau_{2}\right), \\
L_{84}= & \left(Q_{0} \omega_{10}^{*}-Q_{2}\left(\omega_{10}^{*}\right)^{3}\right) \cos \tau_{10}^{*} \omega_{10}^{*}+Q_{1}\left(\omega_{10}^{*}\right)^{2} \sin \tau_{10}^{*} \omega_{10}^{*} \\
& +T_{0} \omega_{10}^{*} \cos \left(\tau_{10}^{*}+\tau_{2}\right)+T_{1}\left(\omega_{10}^{*}\right)^{2} \sin \left(\tau_{10}^{*}+\tau_{2}\right) .
\end{aligned}
$$

Obviously, if $\left(H_{52}\right): L_{81} L_{83}+L_{82} L_{84} \neq 0$ holds,

$$
\operatorname{sign}\left\{\frac{d(\operatorname{Re}(\lambda))}{d \tau_{1}}\right\}_{\tau_{1}=\tau_{10}^{*}}=\operatorname{sign}\left\{\operatorname{Re}\left(\frac{d \lambda}{d \tau_{1}}\right)\right\}_{\tau_{1}=\tau_{10}^{*}} \neq 0
$$

Theorem 4 If $R_{0}>1$ and conditions $\left(H_{1}\right),\left(H_{51}\right)$, and $\left(H_{52}\right)$ hold, then the disease-present equilibrium $E^{*}\left(S^{*}, I^{*}, R^{*}\right)$ of system (2) is locally asymptotically stable when $\tau_{1} \in\left[0, \tau_{10}^{*}\right)$; system (2) undergoes a Hopf bifurcation when $\tau_{1}=\tau_{10}^{*}$ and a family of periodic solutions bifurcate from the disease-present equilibrium $E^{*}\left(S^{*}, I^{*}, R^{*}\right)$ near $\tau_{1}=\tau_{10}^{*}$. 


\section{Global stability of the disease-present equilibrium}

Theorem 5 If $\max \left\{l_{1}, l_{2}, l_{3}\right\}<0$, with

$$
\begin{aligned}
l_{1}= & {\left[\frac{p_{11}+q_{11}}{m_{1}}\left(1+\frac{q_{11} M_{1} \tau_{1}}{m_{1}}+\frac{q_{21} M_{1} \tau_{1}}{m_{2}}\right)+\frac{q_{21}}{m_{2}}\left(1+\frac{M_{2} \tau_{1}}{m_{2}}\left(q_{22}+s_{22}\right)+\frac{s_{32} M_{2} \tau_{1}}{m_{3}}\right)\right.} \\
& \left.+\frac{q_{12} q_{21} M_{2} \tau_{1}}{m_{1} m_{2}}+\frac{p_{31}}{m_{3}}\right]<0, \\
l_{2}= & {\left[\frac{p_{12}+q_{12}}{m_{1}}\left(1+\frac{q_{11} M_{1} \tau_{1}}{m_{1}}+\frac{q_{21} M_{1} \tau_{1}}{m_{2}}\right)\right.} \\
& +\frac{p_{22}+q_{22}+s_{22}}{m_{2}}\left(\frac{q_{22} M_{2} \tau_{1}}{m_{2}}+\frac{s_{22} M_{2} \tau_{2}}{m_{2}}+1\right)+\frac{q_{12} M_{2}}{m_{1} m_{2}}\left(p_{22} \tau_{1}+q_{22} \tau_{1}+s_{22} \tau_{2}\right) \\
& \left.+\frac{s_{32} M_{2}}{m_{2} m_{3}}\left(p_{22} \tau_{2}+q_{22} \tau_{1}+s_{22} \tau_{2}\right)+\frac{p_{32}+s_{32}}{m_{3}}\right]<0, \\
l_{3}= & {\left[\frac{p_{13}}{m_{1}}+\frac{p_{33}}{m_{3}}+\frac{p_{13} M_{1} \tau_{1}}{m_{1}}\left(\frac{q_{11}}{m_{1}}+\frac{q_{21}}{m_{2}}\right)\right]<0, }
\end{aligned}
$$

where $m_{1}<S(t)<M_{1}, m_{2}<I(t)<M_{2}$, and $m_{3}<R(t)<M_{3}$ for $t>0$, the disease-present equilibrium $E^{*}$ of system (2) is globally asymptotically stable.

Proof Let $S(t)=S^{*} e^{x(t)}, I(t)=I^{*} e^{y(t)}, R(t)=R^{*} e^{z(t)}$. Then $E^{*}\left(S^{*}, I^{*}, R^{*}\right)$ becomes the trivial equilibrium for $x(t)=y(t)=z(t)=0$ for all $t>0$, and system (4) can be reduced in the following form:

$$
\begin{aligned}
\frac{d x}{d t}= & \frac{p_{11} S^{*}}{S}\left[e^{x(t)}-1\right]+\frac{p_{12} I^{*}}{S}\left[e^{y(t)}-1\right]+\frac{p_{13} R^{*}}{S}\left[e^{z(t)}-1\right] \\
& +\frac{q_{11} S^{*}}{S}\left[e^{x\left(t-\tau_{1}\right)}-1\right]+\frac{q_{12} I^{*}}{S}\left[e^{y\left(t-\tau_{1}\right)}-1\right], \\
\frac{d y}{d t}= & \frac{p_{22} I^{*}}{I}\left[e^{y(t)}-1\right]+\frac{q_{21} S^{*}}{I}\left[e^{x\left(t-\tau_{1}\right)}-1\right]+\frac{q_{22} I^{*}}{I}\left[e^{y\left(t-\tau_{1}\right)}-1\right] \\
& +\frac{s_{22} I^{*}}{I}\left[e^{y\left(t-\tau_{2}\right)}-1\right], \\
\frac{d z}{d t}= & \frac{p_{31} S^{*}}{R}\left[e^{x(t)}-1\right]+\frac{p_{32} I^{*}}{R}\left[e^{y(t)}-1\right]+\frac{p_{33} R^{*}}{R}\left[e^{z(t)}-1\right] \\
& +\frac{s_{32} I^{*}}{R}\left[e^{y\left(t-\tau_{2}\right)}-1\right] .
\end{aligned}
$$

Now, we have

$$
\begin{aligned}
& e^{x\left(t-\tau_{1}\right)}=e^{x(t)}-\int_{t-\tau_{1}}^{t} e^{x(\omega)} \frac{d x}{d \omega} d \omega, \\
& e^{y\left(t-\tau_{1}\right)}=e^{y(t)}-\int_{t-\tau_{1}}^{t} e^{y(\omega)} \frac{d y}{d \omega} d \omega .
\end{aligned}
$$

Now, by using Eq. (23), Eq. (26), and Eq. (27), we have

$$
\begin{aligned}
\frac{d x}{d t}= & \frac{p_{11} S^{*}}{S}\left[e^{x(t)}-1\right]+\frac{p_{12} I^{*}}{S}\left[e^{y(t)}-1\right]+\frac{p_{13} R^{*}}{S}\left[e^{z(t)}-1\right] \\
& +\frac{q_{11} S^{*}}{S}\left[e^{x(t)}-1\right]-\frac{q_{11} S^{*}}{S} \int_{t-\tau_{1}}^{t} e^{x(\omega)} \frac{d x}{d \omega} d \omega
\end{aligned}
$$


Zou et al. Advances in Difference Equations

(2020) 2020:351

Page 13 of 30

$$
\begin{aligned}
& +\frac{q_{12} I^{*}}{S}\left[e^{y(t)}-1\right]-\frac{q_{12} I^{*}}{S} \int_{t-\tau_{1}}^{t} e^{y(\omega)} \frac{d y}{d \omega} d \omega \\
= & \frac{p_{11} S^{*}}{S}\left[e^{x(t)}-1\right]+\frac{p_{12} I^{*}}{S}\left[e^{y(t)}-1\right]+\frac{p_{13} R^{*}}{S}\left[e^{z(t)}-1\right] \\
& +\frac{q_{11} S^{*}}{S}\left[e^{x(t)}-1\right]+\frac{q_{12} I^{*}}{S}\left[e^{y(t)}-1\right] \\
& -\frac{q_{12} I^{*}}{S} \int_{t-\tau_{1}}^{t} e^{y(\omega)}\left[\frac{p_{22} I^{*}}{I}\left[e^{y(\omega)}-1\right]+\frac{q_{21} S^{*}}{I}\left[e^{x\left(\omega-\tau_{1}\right)}-1\right]\right. \\
& \left.+\frac{q_{22} I^{*}}{I}\left[e^{y\left(\omega-\tau_{1}\right)}-1\right]+\frac{S_{22} I^{*}}{I}\left[e^{y\left(\omega-\tau_{2}\right)}-1\right]\right] d \omega \\
& -\frac{q_{11} S^{*}}{S} \int_{t-\tau_{1}}^{t} e^{x(\omega)}\left[\frac{p_{11} S^{*}}{S}\left[e^{x(\omega)}-1\right]+\frac{p_{12} I^{*}}{S}\left[e^{y(\omega)}-1\right]\right. \\
& \left.+\frac{p_{13} R^{*}}{S}\left[e^{z(\omega)}-1\right]+\frac{q_{11} S^{*}}{S}\left[e^{x\left(\omega-\tau_{1}\right)}-1\right]+\frac{q_{12} I^{*}}{S}\left[e^{y\left(\omega-\tau_{1}\right)}-1\right]\right] d \omega .
\end{aligned}
$$

Let $V_{1}(t)=|x(t)|$,

$$
\begin{aligned}
D^{+} V_{1} \leq & \frac{p_{11} S^{*}}{m_{1}}\left|e^{x(t)}-1\right|+\frac{p_{12} I^{*}}{m_{1}}\left|e^{y(t)}-1\right|+\frac{p_{13} R^{*}}{m_{1}}\left|e^{z(t)}-1\right| \\
& +\frac{q_{11} S^{*}}{m_{1}}\left|e^{x(t)}-1\right|+\frac{q_{12} I^{*}}{m_{1}}\left|e^{y(t)}-1\right| \\
& +\frac{q_{11} S^{*}}{m_{1}} \int_{t-\tau_{1}}^{t} e^{x(\omega)}\left[\frac{p_{11} S^{*}}{m_{1}}\left|e^{x(\omega)}-1\right|+\frac{p_{12} I^{*}}{m_{1}}\left|e^{y(\omega)}-1\right|+\frac{p_{13} R^{*}}{m_{1}}\left|e^{z(\omega)}-1\right|\right. \\
& \left.+\frac{q_{11} S^{*}}{m_{1}}\left|e^{x\left(\omega-\tau_{1}\right)}-1\right|+\frac{q_{12} I^{*}}{m_{1}}\left|e^{y\left(\omega-\tau_{1}\right)}-1\right|\right] d \omega \\
& +\frac{q_{12} I^{*}}{m_{1}} \int_{t-\tau_{1}}^{t} e^{y(\omega)}\left[\frac{p_{22} I^{*}}{m_{2}}\left|e^{y(\omega)}-1\right|+\frac{q_{21} S^{*}}{m_{2}}\left|e^{x\left(\omega-\tau_{1}\right)}-1\right|\right. \\
& \left.+\frac{q_{22} I^{*}}{m_{2}}\left|e^{y\left(\omega-\tau_{1}\right)}-1\right|+\frac{s_{22} I^{*}}{m_{2}}\left|e^{y\left(\omega-\tau_{2}\right)}-1\right|\right] d \omega .
\end{aligned}
$$

We find that there exists $t_{1}>0$ such that $S^{*} e^{x(t)}<M_{1}, I^{*} e^{y(t)}<M_{2}, \forall t>t_{1}$, and for $t>$ $t_{1}+\tau$, where $\tau=\max \left\{\tau_{1}, \tau_{2}\right\}$, we have

$$
\begin{aligned}
D^{+} V_{1} \leq & \frac{\left(p_{11}+q_{11}\right) S^{*}}{m_{1}}\left|e^{x(t)}-1\right|+\frac{\left(p_{12}+q_{12}\right) I^{*}}{m_{1}}\left|e^{y(t)}-1\right|+\frac{p_{13} R^{*}}{m_{1}}\left|e^{z(t)}-1\right| \\
& +\frac{q_{11} M_{1}}{m_{1}} \int_{t-\tau_{1}}^{t}\left[\frac{p_{11} S^{*}}{m_{1}}\left|e^{x(\omega)}-1\right|+\frac{p_{12} I^{*}}{m_{1}}\left|e^{y(\omega)}-1\right|+\frac{p_{13} R^{*}}{m_{1}}|z(\omega)-1|\right. \\
& \left.+\frac{q_{11} S^{*}}{m_{1}}\left|e^{x\left(\omega-\tau_{1}\right)}-1\right|+\frac{q_{12} I^{*}}{m_{1}}\left|e^{y\left(\omega-\tau_{1}\right)}-1\right|\right] d \omega \\
& +\frac{q_{12} M_{2}}{m_{1}} \int_{t-\tau_{1}}^{t}\left[\frac{p_{22} I^{*}}{m_{2}}\left|e^{y(\omega)}-1\right|+\frac{q_{21} S^{*}}{m_{2}}\left|e^{x\left(\omega-\tau_{1}\right)}-1\right|\right. \\
& \left.+\frac{q_{22} I^{*}}{m_{2}}\left|e^{y\left(\omega-\tau_{1}\right)}-1\right|+\frac{s_{22} I^{*}}{m_{2}}\left|e^{y\left(\omega-\tau_{2}\right)}-1\right|\right] d \omega .
\end{aligned}
$$


Due to the form of Eq. (28), we consider the following functional:

$$
\begin{aligned}
\tilde{V}_{1}(t)= & V_{1}(t)+\frac{q_{11} M_{1}}{m_{1}} \int_{t-\tau_{1}}^{t} \int_{h}^{t}\left[\frac{p_{11} S^{*}}{m_{1}}\left|e^{x(\omega)}-1\right|+\frac{p_{12} I^{*}}{m_{1}}\left|e^{y(\omega)}-1\right|+\frac{p_{13} R^{*}}{m_{1}}\left|e^{z(\omega)}-1\right|\right. \\
& \left.+\frac{q_{11} S^{*}}{m_{1}}\left|e^{x\left(\omega-\tau_{1}\right)}-1\right|+\frac{q_{12} I^{*}}{m_{1}}\left|e^{y\left(\omega-\tau_{1}\right)}-1\right|\right] d \omega d h \\
& +\frac{q_{11}^{2} M_{1} S^{*} \tau_{1}}{m_{1}^{2}} \int_{t-\tau_{1}}^{t}\left|e^{x(\omega)}-1\right| d \omega+\frac{q_{11} q_{12} M_{1} I^{*} \tau_{1}}{m_{1}^{2}} \int_{t-\tau_{1}}^{t}\left|e^{y(\omega)}-1\right| d \omega \\
& +\frac{q_{12} M_{2}}{m_{1}} \int_{t-\tau_{1}}^{t} \int_{h}^{t}\left[\frac{p_{22} I^{*}}{m_{2}}\left|e^{y(\omega)}-1\right|+\frac{q_{21} S^{*}}{m_{2}}\left|e^{x\left(\omega-\tau_{1}\right)}-1\right|\right. \\
& \left.+\frac{q_{22} I^{*}}{m_{2}}\left|e^{y\left(\omega-\tau_{1}\right)}-1\right|+\frac{s_{22} I^{*}}{m_{2}}\left|e^{y\left(\omega-\tau_{2}\right)}-1\right|\right] d \omega d h \\
& +\frac{q_{12} q_{21} M_{2} S^{*} \tau_{1}}{m_{1} m_{2}} \int_{t-\tau_{1}}^{t}\left|e^{x(\omega)}-1\right| d \omega \\
& +\frac{q_{12} q_{22} M_{2} I^{*} \tau_{1}}{m_{1} m_{2}} \int_{t-\tau_{1}}^{t}\left|e^{y(\omega)}-1\right| d \omega+\frac{q_{12} s_{22} M_{2} I^{*} \tau_{2}}{m_{1} m_{2}} \int_{t-\tau_{2}}^{t}\left|e^{y(\omega)}-1\right| d \omega .
\end{aligned}
$$

Thus,

$$
\begin{aligned}
D^{+} \tilde{V}_{1}(t) \leq & D^{+} V_{1}(t)+\frac{q_{11} M_{1} \tau_{1}}{m_{1}}\left[\frac{p_{11} S^{*}}{m_{1}}\left|e^{x(t)}-1\right|+\frac{p_{12} I^{*}}{m_{1}}\left|e^{y(t)}-1\right|+\frac{p_{13} R^{*}}{m_{1}}\left|e^{z(t)}-1\right|\right] \\
& +\frac{q_{11}^{2} M_{1} S^{*} \tau_{1}}{m_{1}^{2}}\left|e^{x(t)}-1\right|+\frac{q_{11} q_{12} M_{1} I^{*} \tau_{1}}{m_{1}^{2}}\left|e^{y(t)}-1\right| \\
& -\left[\frac { q _ { 1 1 } M _ { 1 } } { m _ { 1 } } \int _ { t - \tau _ { 1 } } ^ { t } \left(\frac{p_{11} S^{*}}{m_{1}}\left|e^{x(\omega)}-1\right|+\frac{p_{12} I^{*}}{m_{1}}\left|e^{y(\omega)}-1\right|+\frac{p_{13} R^{*}}{m_{1}}\left|e^{z(\omega)}-1\right|\right.\right. \\
& \left.\left.+\frac{q_{11} S^{*}}{m_{1}}\left|e^{x\left(\omega-\tau_{1}\right)}-1\right|+\frac{q_{12} I^{*}}{m_{1}}\left|e^{y\left(\omega-\tau_{1}\right)}-1\right|\right) d \omega\right] \\
& +\frac{q_{12} p_{22} M_{2} I^{*} \tau_{1}}{m_{1} m_{2}}\left|e^{y(t)}-1\right|+\frac{q_{12} q_{21} M_{2} S^{*} \tau_{1}}{m_{1} m_{2}}\left|e^{x(t)}-1\right| \\
& +\frac{q_{12} q_{22} M_{2} I^{*} \tau_{1}}{m_{1} m_{2}}\left|e^{y(t)}-1\right|+\frac{q_{12} s_{22} M_{2} I^{*} \tau_{2}}{m_{1} m_{2}}\left|e^{y(t)}-1\right| \\
& -\left[\frac { q _ { 1 2 } M _ { 2 } } { m _ { 1 } } \int _ { t - \tau _ { 1 } } ^ { t } \left(\frac{p_{22} I^{*}}{m_{2}}\left|e^{y(\omega)}-1\right|+\frac{q_{21} S^{*}}{m_{2}}\left|e^{x\left(\omega-\tau_{1}\right)}-1\right|\right.\right. \\
& \left.\left.+\frac{q_{22} I^{*}}{m_{2}}\left|e^{y\left(\omega-\tau_{1}\right)}-1\right|+\frac{s_{22} I^{*}}{m_{2}}\left|e^{y\left(\omega-\tau_{2}\right)}-1\right|\right) d \omega\right] .
\end{aligned}
$$

Further,

$$
\begin{aligned}
D^{+} \tilde{V}_{1}(t) \leq & {\left[\frac{\left(p_{11}+q_{11}\right) S^{*}}{m_{1}}+\frac{q_{11}\left(p_{11}+q_{11}\right) M_{1} \tau_{1} S^{*}}{m_{1}^{2}}+\frac{q_{12} q_{21} M_{2} \tau_{1} S^{*}}{m_{1} m_{2}}\right]\left|e^{x(t)}-1\right| } \\
& +\left[\frac{\left(p_{12}+q_{12}\right) I^{*}}{m_{1}}+\frac{q_{11}\left(p_{12}+q_{12}\right) M_{1} \tau_{1} I^{*}}{m_{1}^{2}}+\frac{q_{12}\left(p_{22} \tau_{1}+q_{22} \tau_{1}+s_{22} \tau_{2}\right) M_{2} I^{*}}{m_{1} m_{2}}\right] \\
& \times\left|e^{y(t)}-1\right| \\
& +\left[\frac{p_{13} R^{*}}{m_{1}}+\frac{q_{11} p_{13} R^{*} M_{1} \tau_{1}}{m_{1}^{2}}\right]\left|e^{z(t)}-1\right| .
\end{aligned}
$$


Zou et al. Advances in Difference Equations

(2020) 2020:351

Page 15 of 30

Then

$$
\begin{aligned}
D^{+} \tilde{V}_{1}(t) \leq & {\left[\frac{\left(p_{11}+q_{11}\right) S^{*}}{m_{1}}\left(1+\frac{q_{11} M_{1} \tau_{1}}{m_{1}}\right)+\frac{q_{12} q_{21} M_{2} S^{*} \tau_{1}}{m_{1} m_{2}}\right]\left|e^{x(t)}-1\right| } \\
& +\left[\frac{\left(p_{12}+q_{12}\right) I^{*}}{m_{1}}\left(1+\frac{q_{11} M_{1} \tau_{1}}{m_{1}}\right)+\frac{q_{12}\left(p_{22} \tau_{1}+q_{22} \tau_{1}+s_{22} \tau_{2}\right) M_{2} I^{*}}{m_{1} m_{2}}\right] \\
& \times\left|e^{y(t)}-1\right| \\
& +\left[\frac{p_{13} R^{*}}{m_{1}}\left(1+\frac{q_{11} M_{1} \tau_{1}}{m_{1}}\right)\right]\left|e^{z(t)}-1\right| .
\end{aligned}
$$

Next,

$$
e^{y\left(t-\tau_{2}\right)}=e^{y(t)}-\int_{t-\tau_{2}}^{t} e^{y(\omega)} \frac{d y}{d \omega} d \omega .
$$

Then Eq. (24) can be rewritten as follows:

$$
\begin{aligned}
& \frac{d y}{d t}=\frac{p_{22} I^{*}}{I}\left[e^{y(t)}-1\right]+\frac{q_{21} S^{*}}{I}\left[\left(e^{x(t)}-1\right)-\int_{t-\tau_{1}}^{t} e^{x(\omega)} \frac{d x}{d \omega} d \omega\right] \\
& +\frac{q_{22} I^{*}}{I}\left[\left(e^{y(t)}-1\right)-\int_{t-\tau_{1}}^{t} e^{y(\omega)} \frac{d y}{d \omega} d \omega\right]+\frac{s_{22} I^{*}}{I}\left[\left(e^{y(t)}-1\right)-\int_{t-\tau_{2}}^{t} e^{y(\omega)} \frac{d y}{d \omega} d \omega\right] \\
& =\frac{p_{22} I^{*}}{I}\left[e^{y(t)}-1\right]+\frac{q_{21} S^{*}}{I}\left[e^{x(t)}-1\right]+\frac{q_{22} I^{*}}{I}\left[e^{y(t)}-1\right]+\frac{s_{22} I^{*}}{I}\left[e^{y(t)}-1\right] \\
& -\frac{q_{21} S^{*}}{I} \int_{t-\tau_{1}}^{t} e^{x(\omega)} \frac{d x}{d \omega} d \omega-\frac{q_{22} I^{*}}{I} \int_{t-\tau_{1}}^{t} e^{y(\omega)} \frac{d y}{d \omega} d \omega-\frac{s_{22} I^{*}}{I} \int_{t-\tau_{2}}^{t} e^{y(\omega)} \frac{d y}{d \omega} d \omega \\
& =\frac{\left(p_{22}+q_{22}+s_{22}\right) I^{*}}{I}\left[e^{y(t)}-1\right]+\frac{q_{21} S^{*}}{I}\left[e^{x(t)}-1\right] \\
& -\frac{q_{21} S^{*}}{I} \int_{t-\tau_{1}}^{t} e^{x(\omega)}\left[\frac{p_{11} S^{*}}{S}\left[e^{x(\omega)}-1\right]+\frac{p_{12} I^{*}}{S}\left[e^{y(\omega)}-1\right]+\frac{p_{13} R^{*}}{S}\left[e^{z(\omega)}-1\right]\right. \\
& \left.+\frac{q_{11} S^{*}}{S}\left[e^{x\left(\omega-\tau_{1}\right)}-1\right]+\frac{q_{12} I^{*}}{S}\left[e^{y\left(\omega-\tau_{1}\right)}-1\right]\right] d \omega \\
& -\frac{q_{22} I^{*}}{I} \int_{t-\tau_{1}}^{t} e^{y(\omega)}\left[\frac{p_{22} I^{*}}{I}\left[e^{y(\omega)}-1\right]+\frac{q_{21} S^{*}}{I}\left[e^{x\left(\omega-\tau_{1}\right)}-1\right]+\frac{q_{22} I^{*}}{I}\left[e^{y\left(\omega-\tau_{1}\right)}-1\right]\right. \\
& \left.+\frac{s_{22} I^{*}}{I}\left[e^{y\left(\omega-\tau_{2}\right)}-1\right]\right] d \omega-\frac{s_{22} I^{*}}{I} \int_{t-\tau_{2}}^{t} e^{y(\omega)}\left[\frac{p_{22} I^{*}}{I}\left[e^{y(\omega)}-1\right]\right. \\
& \left.+\frac{q_{21} S^{*}}{I}\left[e^{x\left(\omega-\tau_{1}\right)}-1\right]+\frac{q_{22} I^{*}}{I}\left[e^{y\left(\omega-\tau_{1}\right)}-1\right]+\frac{s_{22} I^{*}}{I}\left[e^{y\left(\omega-\tau_{2}\right)}-1\right]\right] d \omega .
\end{aligned}
$$

Let $V_{2}(t)=|y(t)|$,

$$
\begin{aligned}
D^{+} V_{2} \leq & \frac{\left(p_{22}+q_{22}+s_{22}\right) I^{*}}{m_{2}}\left|e^{y(t)}-1\right|+\frac{q_{21} S^{*}}{m_{2}}\left|e^{x(t)}-1\right| \\
& +\frac{q_{21} S^{*}}{m_{2}} \int_{t-\tau_{1}}^{t} e^{x(\omega)}\left[\frac{p_{11} S^{*}}{m_{1}}\left|e^{x(\omega)}-1\right|+\frac{p_{12} I^{*}}{m_{1}}\left|e^{y(\omega)}-1\right|+\frac{p_{13} R^{*}}{m_{1}}\left|e^{z(\omega)}-1\right|\right. \\
& \left.+\frac{q_{11} S^{*}}{m_{1}}\left|e^{x\left(\omega-\tau_{1}\right)}-1\right|+\frac{q_{12} I^{*}}{m_{1}}\left|e^{y\left(\omega-\tau_{1}\right)}-1\right|\right] d \omega
\end{aligned}
$$




$$
\begin{aligned}
& +\frac{q_{22} I^{*}}{m_{2}} \int_{t-\tau_{1}}^{t} e^{y(\omega)}\left[\frac{p_{22} I^{*}}{m_{2}}\left|e^{y(\omega)}-1\right|+\frac{q_{21} S^{*}}{m_{2}}\left|e^{x\left(\omega-\tau_{1}\right)}-1\right|\right. \\
& \left.+\frac{q_{22} I^{*}}{m_{2}}\left|e^{y\left(\omega-\tau_{1}\right)}-1\right|+\frac{s_{22} I^{*}}{m_{2}}\left|e^{\omega-\tau_{2}}-1\right|\right] d \omega \\
& +\frac{s_{22} I^{*}}{m_{2}} \int_{t-\tau_{2}}^{t} e^{y(\omega)}\left[\frac{p_{22} I^{*}}{m_{2}}\left|e^{y(\omega)}-1\right|\right. \\
& \left.+\frac{q_{21} S^{*}}{m_{2}}\left|e^{x\left(\omega-\tau_{1}\right)}-1\right|+\frac{q_{22} I^{*}}{m_{2}}\left|e^{y\left(\omega-\tau_{1}\right)}-1\right|+\frac{s_{22} I^{*}}{m_{2}}\left|e^{y\left(\omega-\tau_{2}\right)}-1\right|\right] d \omega .
\end{aligned}
$$

Then, we find that there exists $t_{1}>0$ such that $S^{*} e^{x(t)}<M_{1}, I^{*} e^{y(t)}<M_{2}, \forall t>t_{1}$, and for $t>t_{1}+\tau$, where $\tau=\max \left\{\tau_{1}, \tau_{2}\right\}$, we have

$$
\begin{aligned}
D^{+} V_{2} \leq & \frac{\left(p_{22}+q_{22}+s_{22}\right) I^{*}}{m_{2}}\left|e^{y(t)}-1\right|+\frac{q_{21} S^{*}}{m_{2}}\left|e^{x(t)}-1\right| \\
& +\frac{q_{21} M_{1}}{m_{2}} \int_{t-\tau_{1}}^{t}\left[\frac{p_{11} S^{*}}{m_{1}}\left|e^{x(\omega)}-1\right|+\frac{p_{12} I^{*}}{m_{1}}\left|e^{y(\omega)}-1\right|+\frac{p_{13} R^{*}}{m_{1}}\left|e^{z(\omega)}-1\right|\right. \\
& \left.+\frac{q_{11} S^{*}}{m_{1}}\left|e^{x\left(\omega-\tau_{1}\right)}-1\right|+\frac{q_{12} I^{*}}{m_{1}}\left|e^{y\left(\omega-\tau_{1}\right)}-1\right|\right] d \omega+\frac{q_{22} M_{2}}{m_{2}} \int_{t-\tau_{1}}^{t}\left[\frac{p_{22} I^{*}}{m_{2}}\left|e^{y(\omega)}-1\right|\right. \\
& \left.+\frac{q_{21} S^{*}}{m_{2}}\left|e^{x\left(\omega-\tau_{1}\right)}-1\right|+\frac{q_{22} I^{*}}{m_{2}}\left|e^{y\left(\omega-\tau_{1}\right)}-1\right|+\frac{s_{22} I^{*}}{m_{2}}\left|e^{x\left(\omega-\tau_{2}\right)}-1\right|\right] d \omega \\
& +\frac{s_{22} M_{2}}{m_{2}} \int_{t-\tau_{2}}^{t}\left[\frac{p_{22} I^{*}}{m_{2}}\left|e^{y(\omega)}-1\right|+\frac{q_{21} S^{*}}{m_{2}}\left|e^{x\left(\omega-\tau_{1}\right)}-1\right|+\frac{q_{22} I^{*}}{m_{2}}\left|e^{y\left(\omega-\tau_{1}\right)}-1\right|\right. \\
& \left.+\frac{s_{22} I^{*}}{m_{2}}\left|e^{y\left(\omega-\tau_{2}\right)}-1\right|\right] d \omega .
\end{aligned}
$$

Again due to the form of Eq. (31), we consider the following Lyapunov functional:

$$
\begin{aligned}
\tilde{V}_{2}(t)= & V_{2}(t)+\frac{q_{21} M_{1}}{m_{2}} \int_{t-\tau_{1}}^{t} \int_{k}^{t}\left[\frac{p_{11} s^{*}}{m_{1}}\left|e^{x(\omega)}-1\right|+\frac{p_{12} I^{*}}{m_{1}}\left|e^{y(\omega)}-1\right|+\frac{p_{13} R^{*}}{m_{1}}\left|e^{z(\omega)}-1\right|\right. \\
& \left.+\frac{q_{11} S^{*}}{m_{1}}\left|e^{x\left(\omega-\tau_{1}\right)}-1\right|+\frac{q_{12} I^{*}}{m_{1}}\left|e^{y\left(\omega-\tau_{1}\right)}-1\right|\right] d \omega d k \\
& +\frac{q_{11} q_{21} S^{*} M_{1} \tau_{1}}{m_{1} m_{2}} \int_{t-\tau_{1}}^{t}\left|e^{x(\omega)}-1\right| d \omega+\frac{q_{12} q_{21} M_{1} I^{*} \tau_{1}}{m_{1} m_{2}} \int_{t-\tau_{1}}^{t}\left|e^{y(\omega)}-1\right| d \omega \\
& +\frac{q_{22} M_{2}}{m_{2}} \int_{t-\tau_{1}}^{t} \int_{k}^{t}\left[\frac{p_{22} I^{*}}{m_{2}}\left|e^{y(\omega)}-1\right|+\frac{q_{21} S^{*}}{m_{2}}\left|e^{x\left(\omega-\tau_{1}\right)}-1\right|+\frac{q_{22} I^{*}}{m_{2}}\left|e^{y\left(\omega-\tau_{1}\right)}-1\right|\right. \\
& \left.+\frac{s_{22} I^{*}}{m_{2}}\left|e^{y\left(\omega-\tau_{2}\right)}-1\right|\right] d \omega d k+\frac{q_{21} q_{22} S^{*} M_{2} \tau_{1}}{m_{2}^{2}} \int_{t-\tau_{1}}^{t}\left|e^{x(\omega)}-1\right| d \omega \\
& +\frac{q_{22}^{2} I^{*} M_{2} \tau_{1}}{m_{2}^{2}} \int_{t-\tau_{1}}^{t}\left|e^{y(\omega)}-1\right| d \omega+\frac{q_{22} s_{22} M_{2} I^{*} \tau_{2}}{m_{2}^{2}} \int_{t-\tau_{2}}^{t}\left|e^{e^{y(\omega)}}-1\right| d \omega \\
& +\frac{s_{22} M_{2}}{m_{2}} \int_{t-\tau_{2}}^{t} \int_{k}^{t}\left[\frac{p_{22} I^{*}}{m_{2}}\left|e^{y(\omega)}-1\right|+\frac{q_{21} S^{*}}{m_{2}}\left|e^{x\left(\omega-\tau_{1}\right)}-1\right|+\frac{q_{22} I^{*}}{m_{2}}\left|e^{y\left(\omega-\tau_{1}\right)}-1\right|\right. \\
& \left.+\frac{s_{22} I^{*}}{m_{2}}\left|e^{y\left(\omega-\tau_{2}\right)}-1\right|\right] d \omega d k+\frac{q_{21} s_{22} M_{2} S^{*} \tau_{1}}{m_{2}^{2}} \int_{t-\tau_{1}}^{t}\left|e^{x(\omega)}-1\right| d \omega \\
& +\frac{s_{22} q_{22} M_{2} I^{*} \tau_{1}}{m_{2}^{2}} \int_{t-\tau_{1}}^{t}\left|e^{y(\omega)}-1\right| d \omega+\frac{s_{22}^{2} M_{2} I^{*} \tau_{2}}{m_{2}^{2}} \int_{t-\tau_{2}}^{t}\left|e^{y(\omega)}-1\right| d \omega .
\end{aligned}
$$


Thus,

$$
\begin{aligned}
& D^{+} \tilde{V}_{2}(t) \leq D^{+} V_{2}(t)+\left[\frac{q_{21} M_{1} \tau_{1}}{m_{2}}\left(\frac{p_{11} S^{*}}{m_{1}}\left|e^{x(t)}-1\right|+\frac{p_{12} I^{*}}{m_{1}}\left|e^{y(t)}-1\right|+\frac{p_{13} R^{*}}{m_{1}}\left|e^{z(t)}-1\right|\right)\right. \\
&\left.+\frac{q_{11} q_{12} S^{*} M_{1} \tau_{1}}{m_{1} m_{2}}\left|e^{x(t)}-1\right|+\frac{q_{12} q_{21} M_{1} I^{*} \tau_{1}}{m_{1} m_{2}}\left|e^{y(t)}-1\right|\right] \\
&-\left[\frac { q _ { 2 1 } M _ { 1 } } { m _ { 2 } } \int _ { t - \tau _ { 1 } } ^ { t } \left(\frac{p_{11} S^{*}}{m_{1}}\left|e^{x(\omega)}-1\right|+\frac{p_{12} I^{*}}{m_{1}}\left|e_{1}^{y(\omega)}\right|+\frac{p_{13} R^{*}}{m_{1}}\left|e^{z(\omega)}-1\right|\right.\right. \\
&\left.\left.+\frac{q_{11} S^{*}}{m_{1}}\left|e^{x\left(\omega-\tau_{1}\right)}-1\right|+\frac{q_{12} I^{*}}{m_{1}}\left|e^{y\left(\omega-\tau_{1}\right)}-1\right|\right) d \omega\right]+\frac{p_{22} q_{22} M_{2} I^{*} \tau_{1}}{m_{2}^{2}}\left|e^{y(t)}-1\right| \\
&+\frac{q_{21} q_{22} S^{*} M_{2} \tau_{1}}{m_{2}^{2}}\left|e^{x(t)}-1\right|+\frac{q_{22}^{2} I^{*} M_{2} \tau_{1}}{m_{2}^{2}}\left|e^{y(t)}-1\right|+\frac{q_{22} s_{22} M_{2} I^{*} \tau_{2}}{m_{2}^{2}}\left|e^{y(t)}-1\right| \\
&-\left[\frac { q _ { 2 2 } M _ { 2 } } { M _ { 2 } } \int _ { t - \tau _ { 1 } } ^ { t } \left(\frac{p_{22} I^{*}}{m_{2}}\left|e^{y(\omega)}-1\right|+\frac{q_{21} S^{*}}{m_{2}}\left|e^{x\left(\omega-\tau_{1}\right)}-1\right|+\frac{q_{22} I^{*}}{m_{2}}\left|e^{y\left(\omega-\tau_{1}\right)}-1\right|\right.\right. \\
&\left.\left.+\frac{s_{22} I^{*}}{m_{2}}\left|e^{y\left(\omega-\tau_{2}\right)}-1\right|\right) d \omega\right]+\frac{s_{22} p_{22} M_{2} I^{*} \tau_{2}}{m_{2}^{2}}\left|e^{y(t)}-1\right| \\
&+\frac{q_{21} s_{22} M_{2} S^{*} \tau_{1}}{m_{2}^{2}}\left|e^{x(t)}-1\right|+\frac{s_{22} q_{22} M_{2} I^{*} \tau_{1}}{m_{2}^{2}}\left|e^{y(t)}-1\right| \\
&+\frac{s_{22}^{2} M_{2} I^{*} \tau_{2}}{m_{2}^{2}}\left|e^{y(t)}-1\right|-\left[\frac{s_{22} M_{2}}{m_{2}} \int_{t-\tau_{2}}^{t}\left(\frac{p_{22} I^{*}}{m_{2}}\left|e^{y\left(\omega-\tau_{1}\right)}-1\right|+\frac{q_{22} I^{*}}{m_{2}}\left|e^{y\left(\omega-\tau_{1}\right)}-1\right|+\frac{s_{22} I^{*}}{m_{2}}\left|e^{y\left(\omega-\tau_{2}\right)}-1\right|\right) d \omega\right] . \\
&(33) \\
&
\end{aligned}
$$

Then

$$
\begin{aligned}
D^{+} \tilde{V}_{2}(t) \leq & {\left[\frac{q_{21} S^{*}}{m_{2}}+\frac{q_{21}\left(p_{11}+q_{11}\right) S^{*} M_{1} \tau_{1}}{m_{1} m_{2}}+\frac{q_{21}\left(q_{22}+s_{22}\right) M_{2} S^{*} \tau_{1}}{m_{2}^{2}}\right]\left|e^{x(t)}-1\right| } \\
& +\left[\frac{\left(p_{22}+q_{22}+s_{22}\right) I^{*}}{m_{2}}+\frac{q_{21}\left(p_{12}+q_{12}\right) M_{1} I^{*} \tau_{1}}{m_{1} m_{2}}+\frac{q_{22}\left(p_{22}+q_{22}\right) M_{2} I^{*} \tau_{1}}{m_{2}^{2}}\right. \\
& \left.+\frac{q_{22} s_{22} M_{2} I^{*} \tau_{2}}{m_{2}^{2}}+\frac{s_{22}\left(p_{22}+s_{22}\right) M_{2} I^{*} \tau_{2}}{m_{2}^{2}}+\frac{s_{22} q_{22} M_{2} I^{*} \tau_{1}}{m_{1}}\right]\left|e^{y(t)}-1\right| \\
& +\frac{p_{13} q_{21} R^{*} M_{1} \tau_{1}}{m_{1} m_{2}}\left|e^{z(t)}-1\right| \\
= & \frac{q_{21} S^{*}}{m_{2}}\left[1+\frac{M_{1} \tau_{1}}{m_{1}}\left(p_{11}+q_{11}\right)+\frac{M_{2} \tau_{2}}{m_{2}}\left(q_{22}+s_{22}\right)\right]\left|e^{x(t)}-1\right| \\
& +\frac{I^{*}}{m_{2}}\left[\left(p_{22}+q_{22}+s_{22}\right)+\frac{q_{21} M_{1} \tau_{1}\left(p_{12}+q_{12}\right)}{m_{1}}+\frac{q_{22}\left(p_{22}+q_{22}+s_{22}\right) M_{2} \tau_{2}}{m_{2}}\right. \\
& \left.+\frac{s_{22}\left(p_{22}+q_{22}+s_{22}\right) M_{2} \tau_{2}}{m_{2}}\right]\left|e^{y(t)}-1\right|+\frac{p_{13} q_{21} R^{*} M_{1} \tau_{1}}{m_{1} m_{2}}\left|e^{z(t)}-1\right| .
\end{aligned}
$$

Next, Eq. (25) can be rewritten as the following form:

$$
\begin{aligned}
\frac{d z}{d t}= & \frac{p_{31} S^{*}}{R}\left[e^{x(t)}-1\right]+\frac{p_{32} I^{*}}{R}\left[e^{y(t)}-1\right]+\frac{p_{33} R^{*}}{R}\left[e^{z(t)}-1\right] \\
& +\frac{s_{32} I^{*}}{R}\left[e^{y(t)}-1\right]-\frac{s_{32} I^{*}}{R} \int_{t-\tau_{2}}^{t} e^{y(\omega)} \frac{d y}{d \omega} d \omega
\end{aligned}
$$




$$
\begin{aligned}
= & \frac{p_{31} S^{*}}{R}\left[e^{x(t)}-1\right]+\frac{\left(p_{32}+s_{32}\right) I^{*}}{R}\left[e^{y(t)}-1\right]+\frac{p_{33} R^{*}}{R}\left[e^{z(t)}-1\right] \\
& -\frac{s_{32} I^{*}}{R}\left[\int _ { t - \tau _ { 2 } } ^ { t } e ^ { y ( \omega ) } \left(\frac{p_{22} I^{*}}{I}\left(e^{y(\omega)}-1\right)+\frac{q_{21} S^{*}}{I}\left(e^{x\left(\omega-\tau_{1}\right)}-1\right)\right.\right. \\
& \left.\left.+\frac{q_{22} I^{*}}{I}\left(e^{y\left(\omega-\tau_{1}\right)}-1\right)+\frac{s_{22} I^{*}}{I}\left(e^{y\left(\omega-\tau_{2}\right)}-1\right)\right) d \omega\right] .
\end{aligned}
$$

Let $V_{3}(t)=|z(t)|$.

$$
\begin{aligned}
D^{+} V_{3} \leq & \frac{p_{31} S^{*}}{m_{3}}\left|e^{x(t)}-1\right|+\frac{\left(p_{32}+s_{32}\right) I^{*}}{m_{3}}\left|e^{y(t)}-1\right|+\frac{p_{33} R^{*}}{m_{3}}\left|e^{z(t)}-1\right| \\
& +\frac{s_{32} I^{*}}{m_{3}} \int_{t-\tau_{2}}^{t} e^{y(\omega)}\left[\frac{p_{22} I^{*}}{m_{2}}\left|e^{y(\omega)}-1\right|+\frac{q_{21} S^{*}}{m_{2}}\left|e^{x\left(\omega-\tau_{1}\right)}-1\right|\right. \\
& \left.+\frac{q_{22} I^{*}}{m_{2}}\left|e^{y\left(\omega-\tau_{1}\right)}-1\right|+\frac{s_{22} I^{*}}{m_{2}}\left|e^{y\left(\omega-\tau_{2}\right)}-1\right|\right] d \omega .
\end{aligned}
$$

Then we find that there exists $t_{1}>0$ such that $I^{*} e^{y(\omega)}<M_{2}, \forall t>t_{1}$, and for $t>t+\tau$, $\tau=\max \left\{\tau_{1}, \tau_{2}\right\}$. We have

$$
\begin{aligned}
D^{+} V_{3} \leq & \frac{p_{31} S^{*}}{m_{3}}\left|e^{x(t)}-1\right|+\frac{\left(p_{32}+s_{32}\right) I^{*}}{m_{3}}\left|e^{y(t)}-1\right|+\frac{p_{33} R^{*}}{m_{3}}\left|e^{z(t)}-1\right| \\
& +\frac{s_{32} M_{2}}{m_{3}} \int_{t-\tau_{2}}^{t}\left[\frac{p_{22} I^{*}}{m_{2}}\left|e^{y(\omega)}-1\right|+\frac{q_{21} S^{*}}{m_{2}}\left|e^{x\left(\omega-\tau_{1}\right)}-1\right|\right. \\
& \left.+\frac{q_{22} I^{*}}{m_{2}}\left|e^{y\left(\omega-\tau_{1}\right)}-1\right|+\frac{s_{22} I^{*}}{m_{2}}\left|e^{y\left(\omega-\tau_{2}\right)}-1\right|\right] d \omega .
\end{aligned}
$$

Again due to the form of Eq. (35), we consider the following Lyapunov functional, and we have

$$
\begin{aligned}
\tilde{V}_{3}(t)= & V_{3}(t)+\frac{s_{32} M_{2}}{m_{3}} \int_{t-\tau_{2}}^{t} \int_{l}^{t}\left[\frac{p_{22} I^{*}}{m_{2}}\left|e^{y(\omega)}-1\right|+\frac{q_{21} S^{*}}{m_{2}}\left|e^{x\left(\omega-\tau_{1}\right)}-1\right|\right. \\
& \left.+\frac{q_{22} I^{*}}{m_{2}}\left|e^{y\left(\omega-\tau_{1}\right)}-1\right|+\frac{s_{22} I^{*}}{m_{2}}\left|e^{y\left(\omega-\tau_{2}\right)}-1\right|\right] d \omega d l \\
& +\frac{s_{32} q_{21} M_{2} S^{*} \tau_{1}}{m_{2} m_{3}} \int_{t-\tau_{1}}^{t}\left|e^{x(\omega)}-1\right| d \omega+\frac{s_{32} q_{22} M_{2} I^{*} \tau_{1}}{m_{2} m_{3}} \int_{t-\tau_{1}}^{t}\left|e^{y(\omega)}-1\right| d \omega \\
& +\frac{s_{22} s_{32} M_{2} I^{*} \tau_{2}}{m_{2} m_{3}} \int_{t-\tau_{2}}^{t}\left|e^{y(\omega)}-1\right| d \omega .
\end{aligned}
$$

Thus,

$$
\begin{aligned}
\tilde{V}_{3}(t) \leq & D^{+} V_{3}(t)+\frac{s_{32} p_{22} M_{2} I^{*} \tau_{2}}{m_{2} m_{3}}\left|e^{y(t)}-1\right|+\frac{s_{32} q_{21} M_{2} S^{*} \tau_{1}}{m_{2} m_{3}}\left|e^{x(t)}-1\right| \\
& +\frac{s_{32} q_{22} M_{2} I^{*} \tau_{1}}{m_{2} m_{3}}\left|e^{y(t)}-1\right|+\frac{s_{22} s_{32} M_{2} I^{*} \tau_{2}}{m_{2} m_{3}}\left|e^{y(t)}-1\right| \\
& -\frac{s_{32} M_{2}}{m_{3}} \int_{t-\tau_{2}}^{t}\left[\frac{p_{22} I^{*}}{m_{2}}\left|e^{y(\omega)}-1\right|+\frac{q_{21} S^{*}}{m_{2}}\left|e^{x\left(\omega-\tau_{1}\right)}-1\right|\right. \\
& \left.+\frac{q_{22} I^{*}}{m_{2}}\left|e^{y\left(\omega-\tau_{1}\right)}-1\right|+\frac{s_{22} I^{*}}{m_{2}}\left|e^{y\left(\omega-\tau_{2}\right)}-1\right|\right] d \omega .
\end{aligned}
$$


Then we have

$$
\begin{aligned}
\tilde{V}_{3}(t) \leq & {\left[\frac{p_{31} S^{*}}{m_{3}}+\frac{s_{32} q_{21} M_{2} S^{*} \tau_{1}}{m_{2} m_{3}}\right]\left|e^{x(t)}-1\right| } \\
& +\left[\frac{\left(p_{32}+s_{32}\right) I^{*}}{m_{3}}+\frac{s_{32}\left(p_{22} \tau_{2}+q_{22} \tau_{1}+s_{22} \tau_{2}\right) M_{2} I^{*}}{m_{2} m_{3}}\right]\left|e^{y(t)}-1\right| \\
& +\frac{p_{33} R^{*}}{m_{3}}\left|e^{z(t)}-1\right| .
\end{aligned}
$$

Let us define the functional $V(t)=\tilde{V}_{1}(t)+\tilde{V}_{2}(t)+\tilde{V}_{3}(t)$. Then

$$
\begin{aligned}
& D^{+} V(t)=D^{+} \tilde{V}_{1}(t)+D^{+} \tilde{V}_{2}(t)+D^{+} \tilde{V}_{3}(t) \\
& \leq\left[\frac{\left(p_{11}+s_{11}\right) S^{*}}{m_{1}}+\frac{q_{11}\left(p_{11}+q_{11}\right) M_{1} S^{*} \tau_{1}}{m_{1}^{2}}+\frac{q_{12} q_{21} M_{2} S^{*} \tau_{1}}{m_{1} m_{2}}+\frac{q_{21} S^{*}}{m_{2}}\right. \\
& \left.+\frac{q_{21}\left(p_{11}+q_{11}\right) S^{*} M_{1} \tau_{1}}{m_{1} m_{2}}+\frac{q_{21}\left(q_{22}+s_{22}\right) M_{2} \tau_{1}}{m_{2}^{2}}+\frac{p_{31} S^{*}}{m_{3}}+\frac{s_{32} q_{21} M_{2} S^{*} \tau_{1}}{m_{2} m_{3}}\right] \\
& \times\left|e^{x(t)}-1\right| \\
& +\left[\frac{\left(p_{12}+q_{12}\right) I^{*}}{m_{1}}+\frac{q_{11}\left(p_{12}+q_{12}\right) M_{1} I^{*} \tau_{1}}{m_{1}^{2}}+\frac{q_{12}\left(p_{22} \tau_{1}+q_{22} \tau_{1}+s_{22} \tau_{2}\right) M_{2} I^{*}}{m_{1} m_{2}}\right. \\
& +\frac{\left(p_{22}+q_{22}+s_{22}\right) I^{*}}{m_{2}}+\frac{q_{21}\left(p_{12}+q_{12}\right) M_{1} I^{*} \tau_{1}}{m_{1} m_{2}}+\frac{q_{22}\left(p_{22}+q_{22}\right) M_{2} I^{*} \tau_{1}}{m_{2}^{2}} \\
& +\frac{q_{22} s_{22} M_{2} I^{*} \tau_{2}}{m_{2}^{2}}+\frac{s_{22}\left(p_{22}+s_{22}\right) M_{2} I^{*} \tau_{2}}{m_{2}^{2}}+\frac{s_{22} q_{22} M_{2} I^{*} \tau_{1}}{m_{2}^{2}}+\frac{\left(p_{32}+s_{32}\right) I^{*}}{m_{3}} \\
& \left.+\frac{s_{32}\left(p_{22} \tau_{2}+q_{22} \tau_{1}+s_{22} \tau_{2}\right) M_{2} I^{*}}{m_{2} m_{3}}\right]\left|e^{y(t)}-1\right| \\
& +\left[\frac{p_{13} R^{*}}{m_{1}}+\frac{q_{11} p_{13} R^{*} M_{1} \tau_{1}}{m_{1}^{2}}+\frac{p_{13} q_{21} R^{*} M_{1} \tau_{1}}{m_{1} m_{2}}+\frac{p_{33} R^{*}}{m_{3}}\right]\left|e^{z(t)}-1\right| \\
& =\left[\frac{\left(p_{11}+q_{11}\right) S^{*}}{m_{1}}\left(1+\frac{q_{11} M_{1} \tau_{1}}{m_{1}}+\frac{q_{21} M_{1} \tau_{1}}{m_{2}}\right)\right. \\
& +\frac{q_{21} S^{*}}{m_{2}}\left(1+\frac{M_{2} \tau_{1}}{m_{2}}\left(q_{22}+s_{22}\right)+\frac{s_{32} M_{2} \tau_{1}}{m_{3}}\right) \\
& \left.+\frac{q_{12} q_{21} M_{2} S^{*} \tau_{1}}{m_{1} m_{2}}+\frac{p_{31} S^{*}}{m_{3}}\right]\left|e^{x(t)}-1\right| \\
& +\left[\frac{\left(p_{12}+q_{12}\right) I^{*}}{m_{1}}\left(1+\frac{q_{11} M_{1} \tau_{1}}{m_{1}}+\frac{q_{21} M_{1} \tau_{1}}{m_{2}}\right)\right. \\
& +\left(p_{22}+q_{22}+s_{22}\right)\left(\frac{q_{22} M_{2} I^{*} \tau_{1}}{m_{2}^{2}}+\frac{s_{22} M_{2} I^{*} \tau_{2}}{m_{2}^{2}}+\frac{I^{*}}{m_{2}}\right) \\
& +\frac{q_{12} M_{2} I^{*}}{m_{1} m_{2}}\left(p_{22} \tau_{1}+q_{22} \tau_{1}+s_{22} \tau_{2}\right) \\
& \left.+\frac{s_{32} M_{2} I^{*}}{m_{2} m_{3}}\left(p_{22} \tau_{2}+q_{22} \tau_{1}+s_{22} \tau_{2}\right)+\frac{\left(p_{32}+s_{32}\right) I^{*}}{m_{3}}\right]\left|e^{y(t)}-1\right| \\
& +\left[\frac{p_{13} R^{*}}{m_{1}}+\frac{p_{33} R^{*}}{m_{3}}+\frac{p_{13} R^{*} M_{1} \tau_{1}}{m_{1}}\left(\frac{q_{11}}{m_{1}}+\frac{q_{21}}{m_{2}}\right)\right]\left|e^{z(t)}-1\right| .
\end{aligned}
$$


Thus,

$$
\begin{aligned}
D^{+} V(t) \leq & S^{*}\left[\frac{\left(p_{11}+q_{11}\right)}{m_{1}}\left(1+\frac{q_{11} M_{1} \tau_{1}}{m_{1}}+\frac{q_{21} M_{1} \tau_{1}}{m_{2}}\right)\right. \\
& \left.+\frac{q_{21}}{m_{2}}\left(1+\frac{M_{2} \tau_{1}}{m_{2}}\left(q_{22}+s_{22}\right)+\frac{s_{32} M_{2} \tau_{1}}{m_{3}}\right)+\frac{q_{12} q_{21} M_{2} \tau_{1}}{m_{1} m_{3}}+\frac{p_{31}}{m_{3}}\right]\left|e^{x(t)}-1\right| \\
& +I^{*}\left[\frac{\left(p_{12}+q_{12}\right)}{m_{1}}\left(1+\frac{q_{11} M_{1} \tau_{1}}{m_{1}}+\frac{q_{21} M_{1} \tau_{1}}{m_{2}}\right)\right. \\
& +\frac{\left(p_{22}+q_{22}+s_{22}\right)}{m_{2}}\left(\frac{q_{22} M_{2} \tau_{1}}{m_{2}}+\frac{s_{22} M_{2} \tau_{2}}{m_{2}}+1\right) \\
& \left.+\frac{q_{12} M_{2}}{m_{1} m_{2}}\left(p_{22} \tau_{1}+q_{22} \tau_{1}+s_{22} \tau_{2}\right)+\frac{s_{32} M_{2}}{m_{2} m_{3}}\left(p_{22} \tau_{2}+q_{22} \tau_{1}+s_{22} \tau_{2}\right)+\frac{p_{32}+s_{32}}{m_{3}}\right] \\
& \times\left|e^{y(t)}-1\right| \\
& +R^{*}\left[\frac{p_{13}}{m_{1}}+\frac{p_{33}}{m_{3}}+\frac{p_{13} M_{1} \tau_{1}}{m_{1}}\left(\frac{q_{11}}{m_{1}}+\frac{q_{21}}{m_{2}}\right)\right]\left|e^{z(t)}-1\right| .
\end{aligned}
$$

Then

$$
D^{+} V(t) \leq l_{1} S^{*}\left|e^{x(t)}-1\right|+l_{2} I^{*}\left|e^{y(t)}-1\right|+l_{3} R^{*}\left|e^{z(t)}-1\right| .
$$

Since model (4) is positive invariant, for all $t>t_{1}^{*}$, we have

$$
\begin{aligned}
& \bar{S}>S(t)=S^{*} e^{x(t)}>\underline{S}, \\
& \bar{I}>I(t)=I^{*} e^{y(t)}>\underline{I}, \\
& \bar{R}>R(t)=R^{*} e^{z(t)}>\underline{R} .
\end{aligned}
$$

According to the mean value theorem, we have

$$
\begin{aligned}
& S^{*}\left|e^{x(t)}-1\right|=S^{*} e^{\mu_{1}(t)}|x(t)|>m_{1}|x(t)|, \\
& I^{*}\left|e^{y(t)}-1\right|=I^{*} e^{\mu_{1}(t)}|y(t)|>m_{2}|y(t)|, \\
& R^{*}\left|e^{z(t)}-1\right|=R^{*} e^{\mu_{3}(t)}|z(t)|>m_{3}|z(t)| .
\end{aligned}
$$

We know that $S^{*} e^{\mu_{1}(t)}$ lies between $S^{*}$ and $S(t), I^{*} e^{\mu_{2}(t)}$ lies between $I^{*}$ and $I(t)$, and $R^{*} e^{\mu_{3}(t)}$ lies between $R^{*}$ and $R(t)$. Therefore

$$
D^{+} V(t) \leq-l[|x(t)|,|y(t)|,|z(t)|]
$$

where

$$
-l=\max \left\{l_{1} \bar{S}, l_{2} \bar{I}, l_{3} \bar{R}\right\}<0 .
$$

Hence, by using Lyapunov stability theory, the disease-present equilibrium $E^{*}$ of system (2) is globally asymptotically stable. Hence, the proof is completed. 


\section{Direction and stability of Hopf bifurcation}

In this section, we shall derive the explicit formulae that determine the direction and stability of the Hopf bifurcation at the critical value of $\tau_{10}^{*}$ when $\tau_{1}>0$ and $\tau_{2} \in\left[0, \tau_{20}\right)$. We assume that $\tau_{20}^{*}<\tau_{10}^{*}$, where $\tau_{20}^{*} \in\left[0, \tau_{20}\right)$. For convenience, let $\tau_{1}=\tau_{10}^{*}+\varrho(\varrho \in \mathrm{R}), u_{1}=S\left(\tau_{1} t\right)$, $u_{2}=I\left(\tau_{1} t\right), u_{3}=R\left(\tau_{1} t\right)$. System (2) becomes

$$
\dot{u}(t)=L_{\varrho}\left(u_{t}\right)+F\left(\varrho, u_{t}\right)
$$

where $u(t)=\left(u_{1}, u_{2}, u_{3}\right)^{T} \in C=C\left([-1,0], \mathbb{R}^{3}\right)$ and $L_{\varrho}: C \rightarrow \mathbb{R}^{3}$ and $F: \mathbb{R} \times C \rightarrow \mathbb{R}^{3}$ are defined as

$$
L_{\varrho} \phi=\left(\tau_{10}^{*}+\varrho\right)\left(L_{1 \max } \phi(0)+L_{2 \max } \phi\left(-\frac{\tau_{20}^{*}}{\tau_{10}^{*}}\right)+L_{3 \max } \phi(-1)\right),
$$

and

$$
\begin{aligned}
F(\varrho, \phi) & =\left(\tau_{10}^{*}+\varrho\right)\left[F_{1}, F_{2}, F_{2}\right]^{T}, \\
L_{1 \max } & =\left(\begin{array}{ccc}
p_{11} & p_{12} & p_{13} \\
0 & p_{22} & 0 \\
p_{31} & p_{32} & p_{33}
\end{array}\right), \\
L_{3 \max } & =\left(\begin{array}{ccc}
q_{11} & q_{12} & 0 \\
q_{21} & q_{22} & 0 \\
0 & 0 & 0
\end{array}\right),
\end{aligned}
$$

and

$$
\begin{aligned}
F_{1}= & p_{14} \phi_{1}^{2}(0)+p_{15} \phi_{1}(0) \phi_{2}(0)+p_{16} \phi_{1}(0) \phi_{3}(0)+p_{17} \phi_{2}(0) \phi_{3}(0) \\
& +p_{18} \phi_{1}(-1) \phi_{2}(-1)+p_{19} \phi_{2}^{2}(0)+p_{110} \phi_{3}^{2}(0), \\
F_{2}= & p_{23} \phi_{1}(-1) \phi_{2}(-1), \\
F_{3}= & p_{34} \phi_{1}^{2}(0)+p_{35} \phi_{1}(0) \phi_{2}(0)+p_{36} \phi_{1}(0) \phi_{3}(0)+p_{37} \phi_{2}(0) \phi_{3}(0) \\
& +p_{38} \phi_{2}^{2}(0)+p_{39} \phi_{3}^{2}(0),
\end{aligned}
$$

with

$$
\begin{aligned}
& p_{14}=-\frac{b(1-q)}{K}, \quad p_{15}=-\frac{(1-q)(1+r) b}{K}, \quad p_{16}=-\frac{b(1-q)}{K}, \\
& p_{17}=-\frac{(1-q)(1+r) b}{K}, \quad p_{18}=-\beta, \quad p_{19}=\frac{(1-q) b r}{K}, \\
& p_{110}=\frac{b(1-q)}{K}, \quad p_{23}=\beta, \quad p_{34}=-\frac{q b}{K}, \quad p_{35}=-\frac{q b(1+r)}{K}, \\
& p_{36}=-\frac{q b}{K}, \quad p_{37}=-\frac{q b(1+r)}{K}, \quad p_{38}=-\frac{q b r}{K}, \quad p_{39}=-\frac{q b}{K} .
\end{aligned}
$$

Accordingly, there exists $\eta(\theta, \varrho)$ such that

$$
L_{\varrho} \phi=\int_{-1}^{0} d \eta(\theta, \varrho) \phi(\theta)
$$




\section{Choosing}

$$
\eta(\theta, \varrho)= \begin{cases}\left(\tau_{10}^{*}+\varrho\right)\left(L_{1 \max }+L_{2 \max }+L_{3 \max }\right), & \theta=0, \\ \left(\tau_{10}^{*}+\varrho\right)\left(L_{2 \max }+L_{3 \max }\right), & \theta \in\left[-\frac{\tau_{\tau_{10}^{*}}^{*}}{\tau_{10}^{*}}, 0\right), \\ \left(\tau_{10}^{*}+\varrho\right) L_{3 \max }, & \theta \in\left(-1,-\frac{\tau_{20}^{*}}{\tau_{10}^{*}}\right), \\ 0, & \theta=-1 .\end{cases}
$$

For $\phi \in C\left([-1,0], \mathbb{R}^{3}\right)$, define

$$
A(\varrho) \phi= \begin{cases}\frac{d \phi(\theta)}{d \theta}, & -1 \leq \theta<0 \\ \int_{-1}^{0} d \eta(\theta, \varrho) \phi(\theta), & \theta=0\end{cases}
$$

and

$$
R(\varrho) \phi= \begin{cases}0, & -1 \leq \theta<0 \\ F(\varrho, \phi), & \theta=0 .\end{cases}
$$

System (37) becomes

$$
\dot{u}(t)=A(\varrho) u_{t}+R(\varrho) u_{t} .
$$

For $\varphi \in C^{1}\left([0,1],\left(\mathbb{R}^{3}\right)^{*}\right)$, define

$$
A^{*}(\varphi)= \begin{cases}-\frac{d \varphi(s)}{d s}, & 0<s \leq 1, \\ \int_{-1}^{0} d \eta^{T}(s, 0) \varphi(-s), & s=0,\end{cases}
$$

with the bilinear inner form for $A(0)$ and $A^{*}$

$$
\langle\varphi(s), \phi(\theta)\rangle=\bar{\varphi}(0) \phi(0)-\int_{\theta=-1}^{0} \int_{\xi=0}^{\theta} \bar{\varphi}(\xi-\theta) d \eta(\theta) \phi(\xi) d \xi
$$

where $\eta(\theta)=\eta(\theta, 0)$.

Suppose that $V(\theta)=\left(1, V_{2}, V_{3}\right) e^{i \tau_{10}^{*} \omega_{10}^{*} \theta}(\theta \in[-1,0])$ is the eigenvector of $A(0)$ associated with the eigenvalue $+i \omega_{10} \tau_{10}^{*}$ and $V^{*}(s)=D\left(1, V_{2}^{*}, V_{3}^{*}\right) e^{i \tau_{10}^{*} \omega_{10}^{*} s}(s \in[0,1])$ is the eigenvector of $A(0)$ associated with the eigenvalue $-i \omega_{10} \tau_{10}^{*}$. By the definitions of $A(0)$ and $A^{*}$, we can obtain

$$
\begin{aligned}
& V_{2}=\frac{q_{21} e^{-i \tau \tau_{0}^{*} \omega_{10}^{*}}}{i \omega_{10}^{*}-p_{22}-s_{22} e^{-i \tau \tau_{20}^{*}} \omega_{10}^{*}-q_{22} e^{-i \tau \tau_{10}^{*} \omega_{10}^{*}}}, \\
& V_{3}=\frac{i \omega_{10}^{*}-p_{11}-q_{11} e^{-i \tau \tau_{10}^{*} \omega_{10}^{*}}-\left(p_{12}+q_{12} e^{-i \tau_{10}^{*} \omega_{10}^{*}}\right) V_{2}}{p_{13}}, \\
& V_{2}^{*}=-\frac{i \omega_{10}^{*}+p_{11}+q_{11} e^{i \tau \tau_{10}^{*} \omega_{10}^{*}}}{q_{21} e^{i \tau \tau_{10}^{*} \omega_{10}^{*}}} \\
& V_{3}^{*}=-\frac{p_{13}}{i \omega_{10}^{*}+p_{33}} .
\end{aligned}
$$


From Eq. (43), we obtain

$$
\begin{aligned}
\bar{D}= & {\left[1+V_{2} \bar{V}_{2}^{*}+V_{3} \bar{V}_{3}^{*}+\tau_{20}^{*} e^{-i \tau_{10}^{*} \omega_{10}^{*}}\left(s_{22} \bar{V}_{2}^{*}+s_{32} \bar{V}_{3}^{*}\right) V_{2}\right.} \\
& \left.+\tau_{10}^{*} e^{-i \tau \tau_{10}^{*} \omega_{10}^{*}}\left(q_{11}+q_{21} \bar{V}_{2}^{*}\right)+\left(q_{12}+q_{22} \bar{V}_{2}^{*}\right) V_{2}\right]^{-1},
\end{aligned}
$$

then $\left\langle V^{*}, V\right\rangle=1$ and $\left\langle V^{*}, \bar{V}\right\rangle=0$.

In what follows, we can obtain $g_{20}, g_{11}, g_{02}$, and $g_{21}$ by using the algorithms in [41] and a similar process as that in [42-44]:

$$
\begin{aligned}
& g_{20}=2 \tau_{10}^{*} \bar{D}\left[p_{14}+p_{15} V_{2}+p_{15} V_{3}+p_{16} V_{2} V_{3}+p_{17} V_{2} V_{3}+p_{18} V_{2} e^{-2 i \tau_{10}^{*} \omega_{10}^{*}}\right. \\
& +p_{19} V_{2}^{2}+P_{110} V_{3}^{2}+p_{23} \bar{V}_{2}^{*} V_{2} e^{-2 i \tau_{10}^{*} \omega_{10}^{*}}+\bar{V}_{3}^{*}\left(p_{34}+p_{35} V_{2}+p_{36} V_{3}\right. \\
& \left.\left.+p_{37} V_{2} V_{3}+p_{38} V_{2}^{2}+p_{39} V_{3}^{2}\right)\right] \text {, } \\
& g_{11}=\tau_{10}^{*} \bar{D}\left[2 p_{14}+p_{15}\left(V_{2}+\bar{V}_{2}\right)+p_{16}\left(V_{3}+\bar{V}_{3}\right)+p_{17}\left(V_{2} \bar{V}_{3}+\bar{V}_{2} V_{3}\right)\right. \\
& +p_{18}\left(V_{2}+\bar{V}_{2}\right)+2 p_{19} V_{2} \bar{V}_{2}+2 p_{110} V_{3} \bar{V}_{3}+p_{23} \bar{V}_{2}^{*}\left(V_{2}+\bar{V}_{2}\right) \\
& +\bar{V}_{3}^{*}\left(2 p_{34}+p_{35}\left(V_{2}+\bar{V}_{2}\right)+p_{36}\left(V_{3}+\bar{V}_{3}\right)+p_{37}\left(V_{2} \bar{V}_{3}+\bar{V}_{2} V_{3}\right)\right. \\
& \left.\left.+2 p_{38} V_{2} \bar{V}_{2}+2 p_{39} V_{3} \bar{V}_{3}\right)\right] \\
& g_{02}=2 \tau_{10}^{*} \bar{D}\left[p_{14}+p_{15} \bar{V}_{2}+p_{15} \bar{V}_{3}+p_{16} \bar{V}_{2} \bar{V}_{3}+p_{17} \bar{V}_{2} \bar{V}_{3}+p_{18} \bar{V}_{2} e^{2 i \tau_{10}^{*} \omega_{10}^{*}}\right. \\
& +p_{19} \bar{V}_{2}^{2}+P_{110} \bar{V}_{3}^{2}+p_{23} \bar{V}_{2}^{*} \bar{V}_{2} e^{2 i \tau_{10}^{*} \omega_{10}^{*}}+\bar{V}_{3}^{*}\left(p_{34}+p_{35} \bar{V}_{2}+p_{36} \bar{V}_{3}\right. \\
& \left.\left.+p_{37} \bar{V}_{2} \bar{V}_{3}+p_{38} \bar{V}_{2}^{2}+p_{39} \bar{V}_{3}^{2}\right)\right] \\
& g_{21}=2 \tau_{10}^{*} \bar{D}\left\{p_{14}\left(2 W_{11}^{(1)}(0)+W_{20}^{(1)}(0)\right)+p_{15}\left(W_{11}^{(1)}(0) V_{2}+\frac{1}{2} W_{20}^{(1)}(0) \bar{V}_{2}\right.\right. \\
& \left.+W_{11}^{(2)}(0)+\frac{1}{2} W_{20}^{(2)}(0)\right)+p_{16}\left(W_{11}^{(1)}(0) V_{3}+\frac{1}{2} W_{20}^{(1)}(0) \bar{V}_{3}\right. \\
& \left.+W_{11}^{(3)}(0)+\frac{1}{2} W_{20}^{(3)}(0)\right)+p_{17}\left(W_{11}^{(2)}(0) V_{3}+\frac{1}{2} W_{20}^{(2)}(0) \bar{V}_{3}\right. \\
& \left.+W_{11}^{(3)}(0) V_{2}+\frac{1}{2} W_{20}^{(3)}(0) \bar{V}_{2}\right)+p_{18}\left(W_{11}^{(1)}(-1) V_{2} e^{-i \tau_{10}^{*} \omega_{10}^{*}}\right. \\
& \left.+\frac{1}{2} W_{20}^{(1)}(-1) \bar{V}_{2} e^{i \tau_{10}^{*} \omega_{10}^{*}}+W_{11}^{(2)}(-1) e^{-i \tau \tau_{10}^{*} \omega_{10}^{*}}+\frac{1}{2} W_{20}^{(2)}(-1) e^{i \tau_{10}^{*} \omega_{10}^{*}}\right) \\
& +p_{19}\left(2 W_{11}^{(2)}(0)+W_{20}^{(2)}(0)\right)+p_{110}\left(2 W_{11}^{(3)}(0)+W_{20}^{(3)}(0)\right) \\
& +p_{23} \bar{V}_{2}^{*}\left(W_{11}^{(1)}(-1) V_{2} e^{-i \tau_{10}^{*} \omega_{10}^{*}}+\frac{1}{2} W_{20}^{(1)}(-1) \bar{V}_{2} e^{i \tau_{10}^{*} \omega_{10}^{*}}\right. \\
& \left.+W_{11}^{(2)}(-1) e^{-i \tau_{10}^{*} \omega_{10}^{*}}+\frac{1}{2} W_{20}^{(2)}(-1) e^{i \tau \tau_{10}^{*} \omega_{10}^{*}}\right)+\bar{V}_{3}^{*}\left(p _ { 3 4 } \left(2 W_{11}^{(1)}(0)\right.\right. \\
& \left.+W_{20}^{(1)}(0)\right)+p_{35}\left(W_{11}^{(1)}(0) V_{2}+\frac{1}{2} W_{20}^{(1)}(0) \bar{V}_{2}+W_{11}^{(2)}(0)+\frac{1}{2} W_{20}^{(2)}(0)\right) \\
& +p_{36}\left(W_{11}^{(1)}(0) V_{3}+\frac{1}{2} W_{20}^{(1)}(0) \bar{V}_{3}+W_{11}^{(3)}(0)+\frac{1}{2} W_{20}^{(3)}(0)\right) \\
& +p_{37}\left(W_{11}^{(2)}(0) V_{3}+\frac{1}{2} W_{20}^{(2)}(0) \bar{V}_{3}+W_{11}^{(3)}(0) V_{2}+\frac{1}{2} W_{20}^{(3)}(0) \bar{V}_{2}\right) \\
& \left.\left.+p_{38}\left(2 W_{11}^{(2)}(0)+W_{20}^{(2)}(0)\right)+p_{39}\left(2 W_{11}^{(3)}(0)+W_{20}^{(3)}(0)\right)\right)\right\}
\end{aligned}
$$


with

$$
\begin{aligned}
& W_{20}(\theta)=\frac{i g_{20} V(0)}{\tau_{10}^{*} \omega_{10}^{*}} e^{i \tau_{10}^{*} \omega_{10}^{*} \theta}+\frac{i \bar{g}_{02} \bar{V}(0)}{3 \tau_{10}^{*} \omega_{10}^{*}} e^{-i \tau_{10}^{*} \omega_{10}^{*} \theta}+E_{1} e^{2 i \tau_{10}^{*} \omega_{10}^{*} \theta}, \\
& W_{11}(\theta)=-\frac{i g_{11} V(0)}{\tau_{10}^{*} \omega_{10}^{*}} e^{i \tau_{10}^{*} \omega_{10}^{*} \theta}+\frac{i \bar{g}_{11} \bar{V}(0)}{\tau_{10}^{*} \omega_{10}^{*}} e^{-i \tau_{10}^{*} \omega_{10}^{*} \theta}+E_{2}
\end{aligned}
$$

with

$$
\begin{aligned}
& E_{1}=2\left(\begin{array}{ccc}
p_{11}^{\prime} & -p_{12}-q_{12} e^{-2 i \omega_{10}^{*} \tau_{10}^{*}} & -p_{13} \\
-q_{21} e^{-2 i \omega_{10}^{*} \tau_{10}^{*}} & p_{22}^{\prime} & 0 \\
-p_{31} & -p_{32}-s_{32} e^{-2 i \omega_{10}^{*} \tau_{20}^{*}} & p_{33}^{\prime}
\end{array}\right)^{-1} \times\left(\begin{array}{c}
E_{1}^{(1)} \\
E_{1}^{(2)} \\
E_{1}^{(3)}
\end{array}\right), \\
& E_{2}=-\left(\begin{array}{ccc}
p_{11}+q_{11} & p_{12}+q_{12} & p_{13} \\
q_{21} & p_{22}+s_{22}+q_{22} & 0 \\
p_{31} & p_{32}+s_{32} & 0
\end{array}\right)^{-1} \times\left(\begin{array}{c}
E_{2}^{(1)} \\
E_{2}^{(2)} \\
E_{2}^{(3)}
\end{array}\right),
\end{aligned}
$$

where

$$
\begin{aligned}
& p_{11}^{\prime}=2 i \omega_{10}^{*}-p_{11}-q_{11} e^{-2 i \omega_{10}^{*} \tau_{10}^{*}}, \\
& p_{22}^{\prime}=2 i \omega_{10}^{*}-p_{22}-s_{22} e^{-2 i \omega_{10}^{*} \tau_{20}^{*}}-q_{22} e^{-2 i \omega_{10}^{*} \tau_{10}^{*},} \\
& p_{33}^{\prime}=2 i \omega_{10}^{*}-p_{33},
\end{aligned}
$$

and

$$
\begin{aligned}
E_{1}^{(1)}= & p_{14}+p_{15} V_{2}+p_{15} V_{3}+p_{16} V_{2} V_{3}+p_{17} V_{2} V_{3}+p_{18} V_{2} e^{-2 i \tau_{10}^{*} \omega_{10}^{*}} \\
& +p_{19} V_{2}^{2}+P_{110} V_{3}^{2} \\
E_{1}^{(2)}= & p_{23} V_{2} e^{-2 i \tau_{10}^{*} \omega_{10}^{*}} \\
E_{1}^{(3)}= & p_{34}+p_{35} V_{2}+p_{36} V_{3}+p_{37} V_{2} V_{3}+p_{38} V_{2}^{2}+p_{39} V_{3}^{2}, \\
E_{2}^{(1)}= & 2 p_{14}+p_{15}\left(V_{2}+\bar{V}_{2}\right)+p_{16}\left(V_{3}+\bar{V}_{3}\right)+p_{17}\left(V_{2} \bar{V}_{3}+\bar{V}_{2} V_{3}\right) \\
& +p_{18}\left(V_{2}+\bar{V}_{2}\right)+2 p_{19} V_{2} \bar{V}_{2}+2 p_{110} V_{3} \bar{V}_{3} \\
E_{2}^{(2)}= & p_{23}\left(V_{2}+\bar{V}_{2}\right), \\
E_{2}^{(3)}= & 2 p_{34}+p_{35}\left(V_{2}+\bar{V}_{2}\right)+p_{36}\left(V_{3}+\bar{V}_{3}\right)+p_{37}\left(V_{2} \bar{V}_{3}+\bar{V}_{2} V_{3}\right) \\
& \left.+2 p_{38} V_{2} \bar{V}_{2}+2 p_{39} V_{3} \bar{V}_{3}\right) .
\end{aligned}
$$

Thus,

$$
\begin{aligned}
& C_{1}(0)=\frac{i}{2 \tau_{10}^{*} \omega_{10}^{*}}\left(g_{11} g_{20}-2\left|g_{11}\right|^{2}-\frac{\left|g_{02}\right|^{2}}{3}\right)+\frac{g_{21}}{2}, \\
& \mu_{2}=-\frac{\operatorname{Re}\left\{C_{1}(0)\right\}}{\operatorname{Re}\left\{\lambda^{\prime}\left(\tau_{10}^{*}\right)\right\}} \\
& \beta_{2}=2 \operatorname{Re}\left\{C_{1}(0)\right\}, \\
& T_{2}=-\frac{\operatorname{Im}\left\{C_{1}(0)\right\}+\mu_{2} \operatorname{Im}\left\{\lambda^{\prime}\left(\tau_{10}^{*}\right)\right\}}{\tau_{10}^{*} \omega_{10}^{*}},
\end{aligned}
$$


in which $\mu_{2}$ determines the direction of the Hopf bifurcation; $\beta_{2}$ determines the stability of bifurcating periodic solutions, and $T_{2}$ determines the period of bifurcating periodic solutions.

\section{Numerical simulation}

Choosing $q=0.15, b=34.45, K=300, r=0.2, \beta=0.087, \mu=2, v=2, \gamma=1.5$. Then system (2) takes the following form:

$$
\left\{\begin{aligned}
\frac{d S(t)}{d t}= & 29.2825\left(1-\frac{S(t)+I(t)+R(t)}{300}\right)(S(t)+0.2 I(t)+R(t)) \\
& -0.087 S\left(t-\tau_{1}\right) I\left(t-\tau_{1}\right)-2 S(t), \\
\frac{d I(t)}{d t}= & 0.087 S\left(t-\tau_{1}\right) I\left(t-\tau_{1}\right)-2 I(t)-1.5 I\left(t-\tau_{2}\right), \\
\frac{d R(t)}{d t}= & 5.1675\left(1-\frac{S(t)+I(t)+R(t)}{300}\right)(S(t)+0.2 I(t)+R(t)) \\
& +1.5 I\left(t-\tau_{2}\right)+2 S(t)-2 R(t),
\end{aligned}\right.
$$

from which we obtain $R_{0}=2.9853>1$ and Eq. (45) becomes

$$
-0.2530\left(I^{*}\right)^{2}+2.7082 I^{*}+1736.3=0 .
$$

Then, the unique disease-present equilibrium $E^{*}(40.2299,88.3672,147.9940)$ can be obtained with the aid of Matlab software package, and assumption $\left(H_{1}\right)$ is satisfied for system (45). Accordingly, system (45) is locally asymptotically stable for $\tau_{1}=\tau_{2}=0$.

For Theorem 1, we can obtain $\omega_{10}=0.3065$ and the critical value $\tau_{10}=1.9565$ for the occurrence of the Hopf bifurcation. Therefore, conditions $\left(H_{21}\right)$ and $\left(H_{22}\right)$ of Theorem 1 hold. Figure 2 implies that the disease-present equilibrium $E^{*}(40.2299,88.3672,147.9940)$ is locally asymptotically stable when $\tau_{1} \in\left[0, \tau_{10}\right)$. Figure 3 reveals that system (45) loses its stability and a Hopf bifurcation appears once the value of $\tau_{1}$ exceeds $\tau_{10}$.

Similarly, we have $\omega_{20}=1.0602$ and $\tau_{20}=1.4775$ for Theorem 2, $\omega_{0}=2.0951$ and $\tau_{0}=$ 0.8805 for Theorem 3, $\omega_{10}^{*}=1.0207$ and $\tau_{10}^{*}=4.0945$ when $\tau_{2}=0.55 \in\left(0, \tau_{20}\right]$ for Theorem 4, respectively. The corresponding numerical simulations can be revealed by Figs. 45, Figs. 6-7, and Figs. 8-9, respectively.
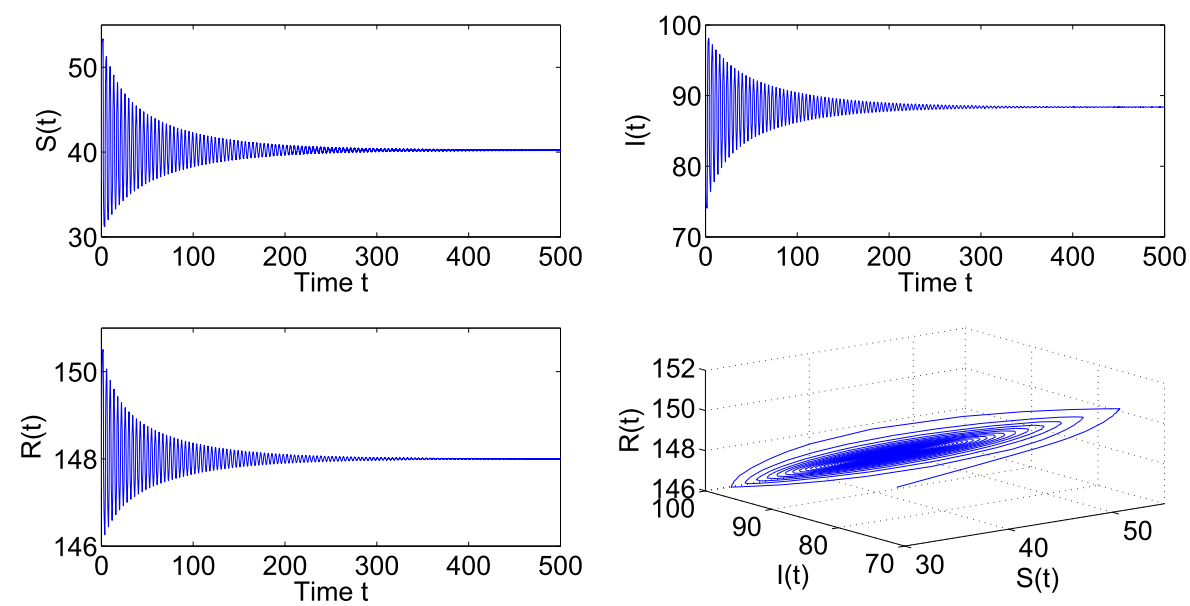

Figure $2 E^{*}$ is locally asymptotically stable when $\tau_{1}=1.850<\tau_{10}=1.9565$ 

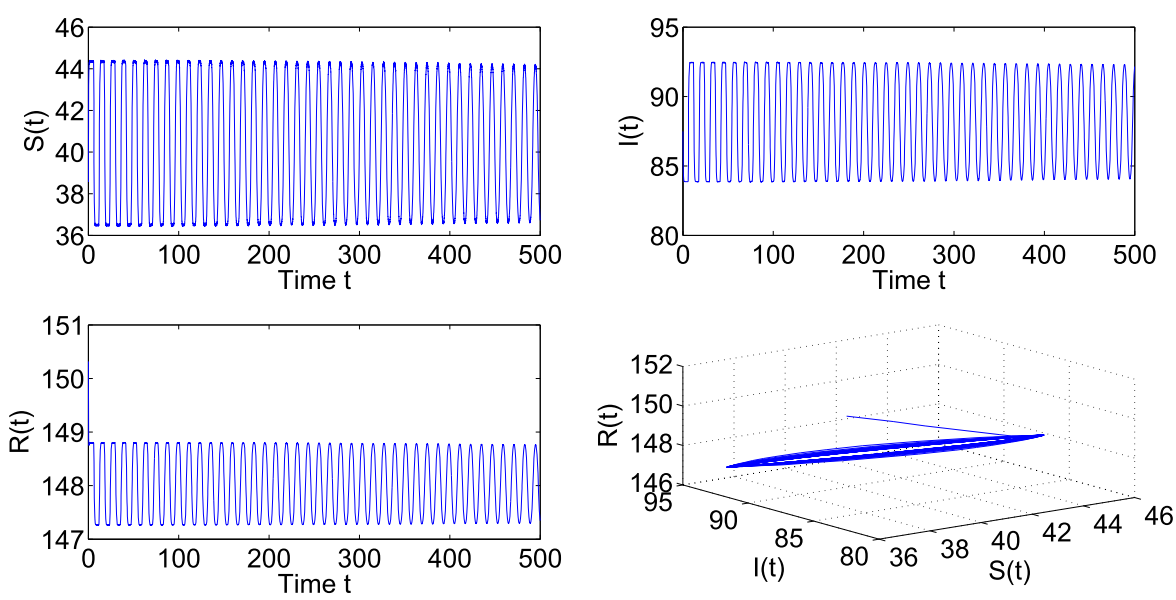

Figure 3 System (45) under goes a Hopf bifurcation when $\tau_{1}=3.750>\tau_{10}=1.9565$
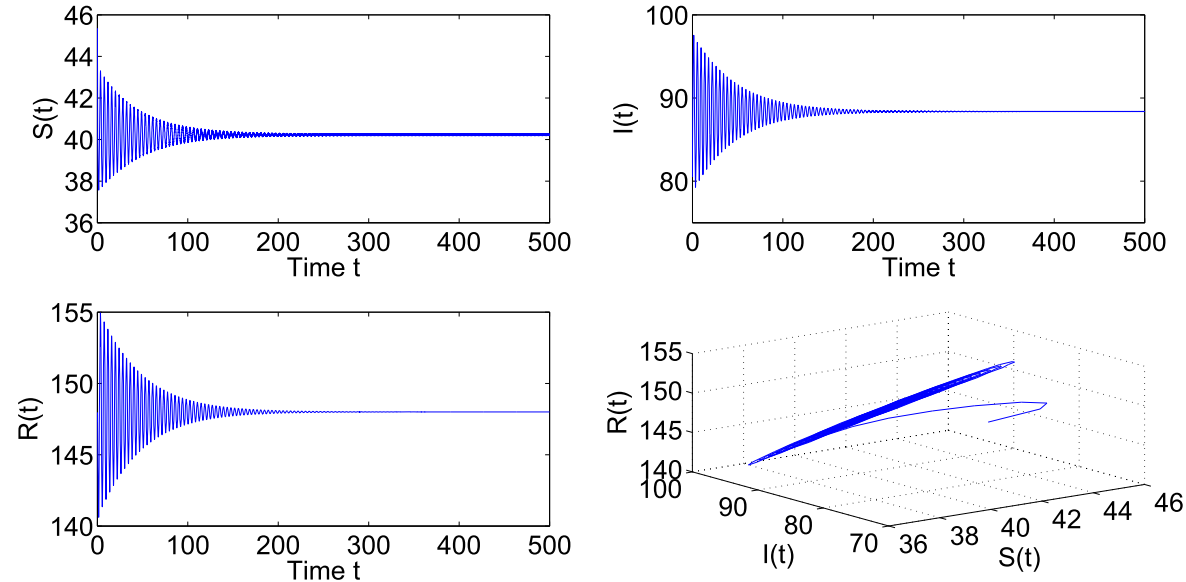

Figure $4 E^{*}$ is locally asymptotically stable when $\tau_{2}=1.326<\tau_{20}=1.4775$
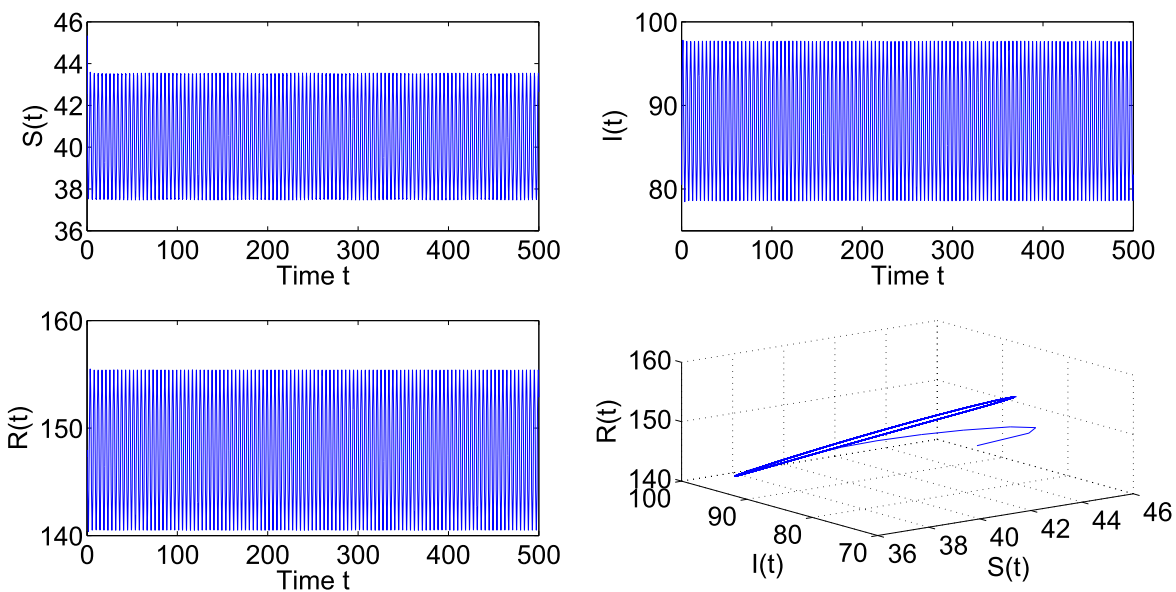

Figure 5 System (45) under goes a Hopf bifurcation when $\tau_{2}=1.518>\tau_{20}=1.4775$ 

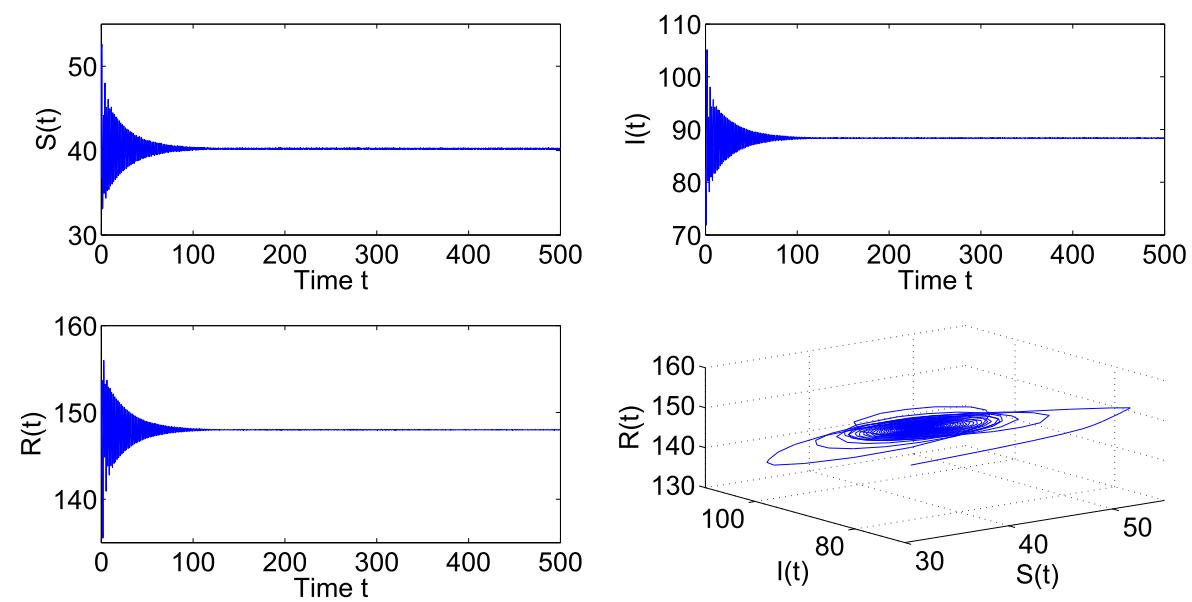

Figure $6 E^{*}$ is locally asymptotically stable when $\tau=0.825<\tau_{0}=0.8805$
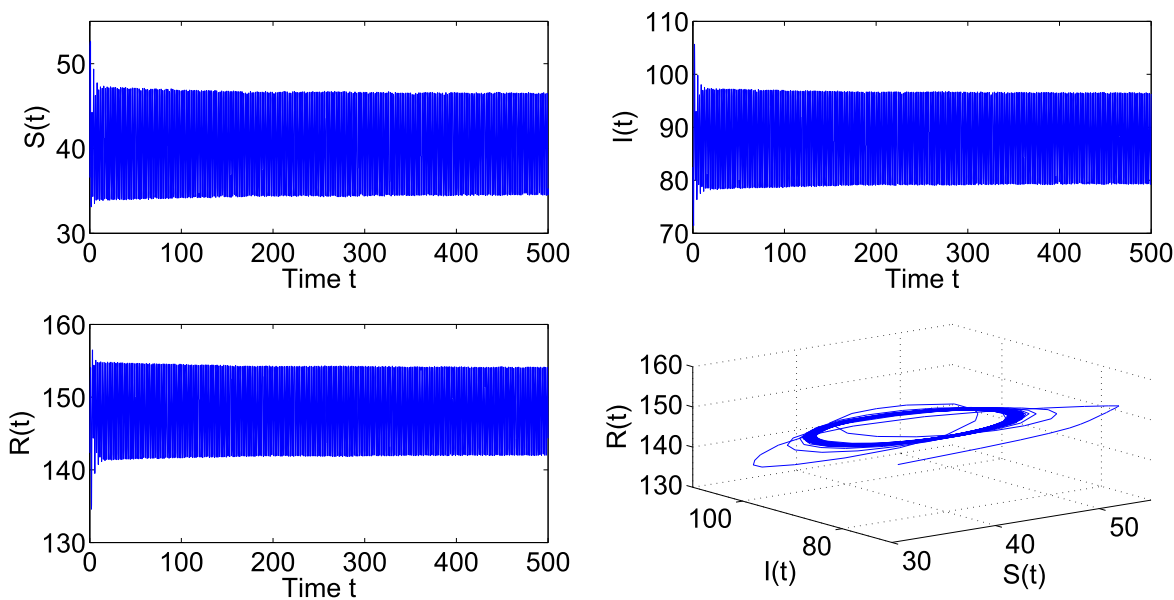

Figure 7 System (45) under goes a Hopf bifurcation when $\tau=0.906>\tau_{0}=0.8805$
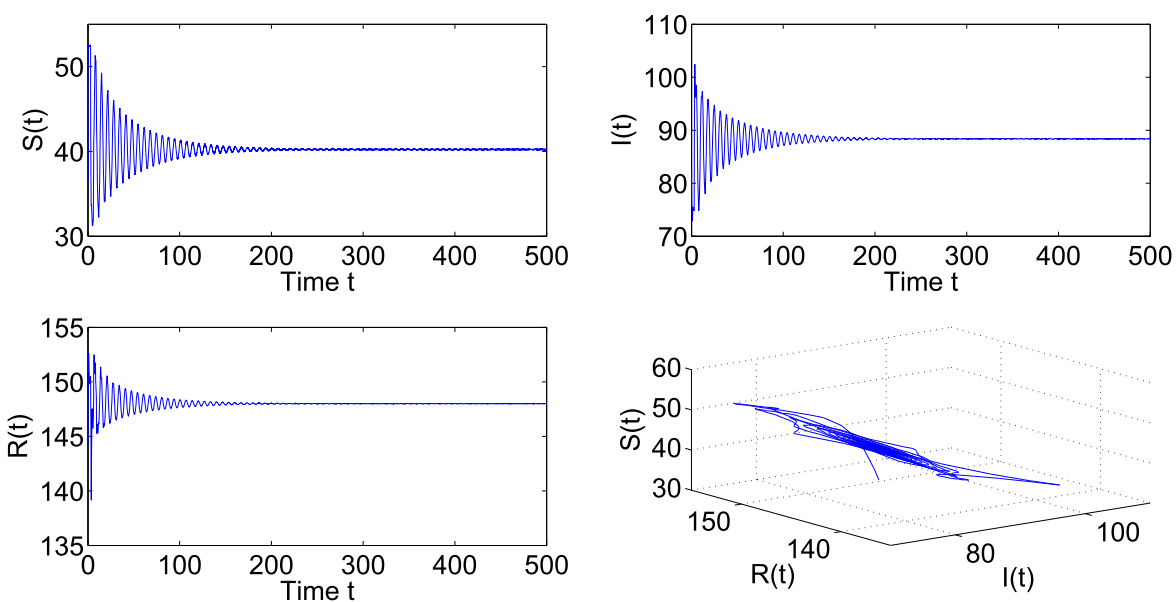

Figure $8 E^{*}$ is locally asymptotically stable when $\tau_{1}=2.895<\tau_{10}^{*}=4.0945$ and $\tau_{2}=0.55 \in\left(0, \tau_{20}\right)$ 

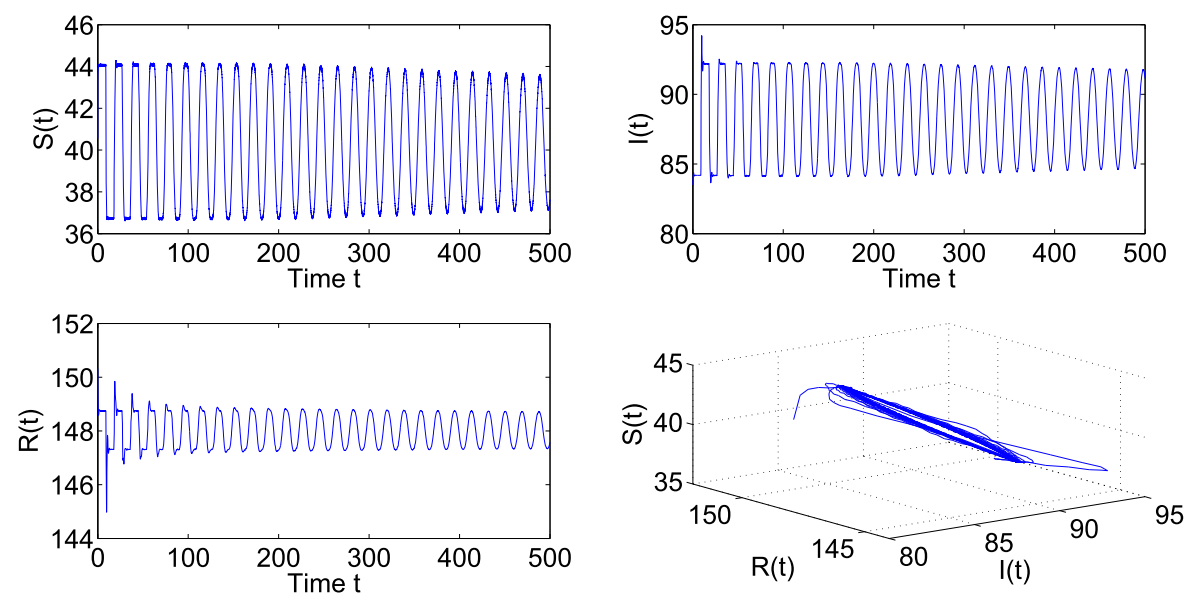

Figure 9 System (45) under goes a Hopf bifurcation when $\tau_{1}=8.896>\tau_{10}^{*}=4.0945$ and $\tau_{2}=0.55 \in\left(0, \tau_{20}\right)$

Specially, we obtain $C_{1}(0)=-37.0955+9.0748 i, \lambda\left(\tau_{10}^{*}\right)=0.6902-1.3033 i$ by some complex computation by means of Matlab package. Based on Eq. (44), we have $\mu_{2}=54.9913$, $\beta_{2}=-74.19100$, and $T_{2}=14.9776$. Since $\mu_{2}>0, \beta_{2}<0$, and $T_{2}>0$, then the Hopf bifurcation is supercritical; the bifurcating periodic solutions are stable and increase.

\section{Conclusions}

A multitude of researchers have paid attention to porcine reproductive and respiratory syndrome due to its vast economic impact on pig production in many countries all over the world. Various strategies at farm level have been developed to combat porcine reproductive and respiratory syndrome. However, it is usually difficult to determine easily which strategy is most suitable in a given farm situation. Mathematical modeling has been extensively used to study and predict the propagation of infectious diseases in populations, and in view of this point, we propose a delayed SIR model for the transmission of PRRSV among a swine population by incorporating two delays into the model formulated in [26]. We derive sufficient conditions to ensure local stability of the disease-present equilibrium and the existence of Hopf bifurcation of the proposed model. Direction and stability of the model are investigated by applying the normal form theory and the center manifold theorem. The obtained findings are justified by computer numerical simulations.

Our findings demonstrate that the model is in an ideal stable state when the value of the two delays is below the corresponding critical value at which the Hopf bifurcation occurs. In this case, we can predict and control the transmission of porcine reproductive and respiratory syndrome virus in a swine population. However, the proposed model will lose its stability and a Hopf bifurcation occurs once the value of the two delays exceeds the corresponding critical value. In this case, the transmission of porcine reproductive and respiratory syndrome virus will be out of control. Thus, it can be concluded that the two delays have an important role on the stability of the model, and our findings in this paper have important theoretical significance and practical value for predicting and controlling the transmission of porcine reproductive and respiratory syndrome virus in a swine population.

The obtained theoretical results in the present paper have shown that the occurrence of the Hopf bifurcation of system (2) is harmful and should be controlled. Therefore, it is an 
interesting issue to investigate Hopf bifurcation control by means of feedback methods or nonlinear time delay feedback methods $[45,46]$. Especially, we are interested in the Hopf bifurcation control issue of fractional-order delayed system (2), which will be our next research work.

\section{Acknowledgements}

The authors express their gratitude to the editors and the referees for their consideration.

\section{Funding}

This research was supported by the Project of Support Program for Excellent Youth Talent in Colleges and Universities of Anhui Province (No. gxyqZD2018044) and the Natural Science Foundation of the Higher Education Institutions of Anhui Province (Nos. KJ2019A0655, KJ2019A0656, KJ2019A0662, KJ2020A0002, KJ2020A0014, KJ2020A0016).

\section{Availability of data and materials}

All of the authors declare that all the data can be accessed in our manuscript in the numerical simulation section.

\section{Competing interests}

The authors declare that there is no conflict of interests.

\section{Authors' contributions}

Each of the authors contributed to each part of this study equally, all authors read and approved the final manuscript.

\section{Author details}

'School of Management Science and Engineering, Anhui University of Finance and Economics, Bengbu, 233030, China. ${ }^{2}$ Department of Mathematics \& Computing, Indian Institute of Technology (Indian School of Mines), Dhanbad, 826004, India. ${ }^{3}$ Department of Mathematics, Alagappa University Alagappapuram, Karaikudi, 630 004, India.

\section{Publisher's Note}

Springer Nature remains neutral with regard to jurisdictional claims in published maps and institutional affiliations.

Received: 12 April 2020 Accepted: 1 July 2020 Published online: 11 July 2020

\section{References}

1. Lambert, M.E., Arsenault, J., Delisle, B., Audet, P., Poljak, Z., D'Allaire, S.: Impact of alignment algorithm on the estimation of pairwise genetic similarity of porcine reproductive and respiratory syndrome virus (PRRSV). BMC Vet. Res. 15, Article ID 135 (2019)

2. Lambert, M.E., Audet, P., Delisle, B., Arsenault, J., D'Allaire, S.: Porcine reproductive and respiratory syndrome virus: web-based interactive tools to support surveillance and control initiatives. Porcine Health Manag. 10, 1-10 (2019)

3. Holtkamp, D.J., Kliebenstein, J.B., Neumann, E.J.: Assessment of the economic impact of porcine reproductive and respiratory syndrome virus on United States pork producers. J. Swine Health Prod. 21, 72-84 (2013)

4. Zou, X.G., Wu, J.X., Hou, G.S., Fan, J., Zhao, L.J., Ou, Y., Zou, H., Zou, Z.Y.: Highly pathogenic porcine reproductive and respiratory syndrome virus cause hyperpyrexia syndrome causes analysis and control measures. Chin. J. Vet. Med. 41, 54-55 (2007) (in Chinese)

5. Lan, H., Zhuo, W.W., Liu, J., Luo, Y., Quan, F.S., Hua, S.: Construction of PRRS GP5-M adenovirus vector and its expression in goat mammary gland. Acta Agric. Boreali-occidentalis Sin. 25, 804-810 (2016) (in Chinese)

6. Xia, W.J., Kundu, S., Maitra, S.: Dynamics of a delayed SEIQ epidemic model. Adv. Differ. Equ. 2018, Article ID 336 (2018)

7. Cheng, Y.L., Lu, D.C., Zhou, J.B., Wei, J.D.: Existence of traveling wave solutions with critical speed in a delayed diffusive epidemic model. Adv. Differ. Equ. 2019, Article ID 494 (2019)

8. Avila, V.E., Perez, A.G.C.: Dynamics of a time-delayed SIR epidemic model with logistic growth and saturated treatment. Chaos Solitons Fractals 127, 55-69 (2019)

9. Kar, T.K., Nandi, S.K., Jana, S.: Stability and bifurcation analysis of an epidemic model with the effect of media. Chaos Solitons Fractals 120, 188-199 (2019)

10. Rihan, F.A., Al-Mdallal, Q.M., Alsakaji, H.J.: A fractional-order epidemic model with time-delay and nonlinear incidence rate. Chaos Solitons Fractals 126, 97-105 (2019)

11. Caraballoa, T., Fatini, M.E., Khalifi, M.E., Gerlachc, R., Pettersson, R.: Analysis of a stochastic distributed delay epidemic model with relapse and gamma distribution kernel. Chaos Solitons Fractals 133, Article ID 109643 (2020)

12. Liu, Y.F., Zhang, Y., Wang, Q.Y.: A stochastic SIR epidemic model with Levy jump and media coverage. Adv. Differ. Equ. 2020, Article ID 70 (2020)

13. Rajasekar, S.P., Pitchaimani, M.: Qualitative analysis of stochastically perturbed SIRS epidemic model with two viruses. Chaos Solitons Fractals 118, 207-221 (2019)

14. Xu, C.Y., Li, X.Y.: The threshold of a stochastic delayed SIRS epidemic model with temporary immunity and vaccination. Chaos Solitons Fractals 111, 227-234 (2018)

15. Khan, T., Khan, A., Zaman, G.: The extinction and persistence of the stochastic hepatitis B epidemic model. Chaos Solitons Fractals 108, 123-128 (2018)

16. Zhao, W.C., Liu, J.L., Chi, M.N., Bian, F.F.: Dynamics analysis of stochastic epidemic models with standard incidence. Adv. Differ. Equ. 2019, Article ID 22 (2019)

17. Akimenko, V.: An age-structured SIR epidemic model with fixed incubation period of infection. Comput. Math. Appl. 73, 1485-1504 (2017) 
18. Li, Y.J., Xu, R., Lin, J.Z.: The stability analysis of an epidemic model with saturating incidence and age-structure in the exposed and infectious classes. Adv. Differ. Equ. 2018, Article ID 202 (2018)

19. Khan, A., Zaman, G.: Global analysis of an age-structured SEIR endemic model. Chaos Solitons Fractals 108, 154-165 (2018)

20. Arruda, A.G., Poljak, Z., Knowles, D., McLean, A.: Development of a stochastic agent-based model to evaluate surveillance strategies for detection of emergent porcine reproductive and respiratory syndrome strains. BMC Vet. Res. 13, Article ID 171 (2017)

21. Jeong, J., Aly, S.S., Cano, J.P., Polson, D., Kass, P.H., Perez, A.M.: Stochastic model of porcine reproductive and respiratory syndrome virus control strategies on a swine farm in the United States. Am. J. Vet. Res. 75, 260-267 (2014)

22. Evans, C.M., Medley, G.F., Creasey, S.J., Green, L.E.: A stochastic mathematical model of the within-herd transmission dynamics of porcine reproductive and respiratory syndrome virus (PRRSV): fade-out and persistence. Prev. Vet. Med. 93, 248-257 (2010)

23. Arruda, A.G., Friendship, R., Carpenter, J., Greer, A., Poljak, Z.: Evaluation of control strategies for porcine reproductive and respiratory syndrome (PRRS) in swine breeding herds using a discrete event agent-based model. PLOS ONE 11, e0166596 (2016)

24. Suksamran, J., Lenbury, Y., Satiracoo, P., Rattanakul, C.: A model for porcine reproductive and respiratory syndrome with time-dependent infection rate: traveling wave solution. Adv. Differ. Equ. 2017, Article ID 215 (2017)

25. Neumann, E.J., James, B.K., Johnson, C.D., John, W.M., Bush, E.J., Seitzinger, A.H., Green, A.L., Zimmerman, J.J.: Assessment of the economic impact of porcine reproductive and respiratory syndrome on swine production in the United States. J. Am. Vet. Med. Assoc. 227, 385-392 (2005)

26. Phoo-ngurn, P., Kiataramkul, C., Chamchod, F.: Modeling the spread of porcine reproductive and respiratory syndrome virus (PRRSV) in a swine population: transmission dynamics, immunity information, and optimal control strategies. Adv. Differ. Equ. 2019, Article ID 432 (2019)

27. Zhang, Z.Z., Kundu, S., Tripathi, J.P., Bugalia, S.: Stability and Hopf bifurcation analysis of an SVEIR epidemic model with vaccination and multiple time delays. Chaos Solitons Fractals 131, Article ID 109483 (2020)

28. Sirijampa, A., Chinviriyasit, S., Chinviriyasit, W.: Hopf bifurcation analysis of a delayed SEIR epidemic model with infectious force in latent and infected period. Adv. Differ. Equ. 2018, Article ID 348 (2018)

29. Jiang, Z.C., Ma, W.B., Wei, J..: Global Hopf bifurcation and permanence of a delayed SEIRS epidemic model. Math. Comput. Simul. 122, 35-54 (2016)

30. Meng, X.Y., Huo, H.F., Zhang, X.B.: Stability and global Hopf bifurcation in a Leslie-Gower predator-prey model with stage structure for prey. J. Appl. Math. Comput. 60, 1-25 (2019)

31. Xiao, Z.W., Xie, X.D., Xue, Y.L.: Stability and bifurcation in a Holling type II predator-prey model with Allee effect and time delay. Adv. Differ. Equ. 2018, Article ID 288 (2018)

32. Kundu, S., Maitra, S.: Dynamics of a delayed predator-prey system with stage structure and cooperation for preys. Chaos Solitons Fractals 114, 453-460 (2018)

33. Meng, X.Y., Qin, N.N., Huo, H.F.: Dynamics analysis of a predator-prey system with harvesting prey and disease in prey species. J. Biol. Dyn. 12, 342-374 (2018)

34. Duan, D.F., Niu, B., Wei, J.J.: Hopf-Hopf bifurcation and chaotic attractors in a delayed diffusive predator-prey model with fear effect. Chaos Solitons Fractals 123, 206-216 (2019)

35. Yang, R.Z., Ma, J.: Analysis of a diffusive predator-prey system with anti-predator behaviour and maturation delay. Chaos Solitons Fractals 109, 128-139 (2018)

36. Xu, C.J., Li, P.L.: Influence of leakage delay on almost periodic solutions for BAM neural networks. IEEE Access 7 , $129741-129757$ (2019)

37. Xu, C.J.: Local and global Hopf bifurcation analysis on simplified bidirectional associative memory neural networks with multiple delays. Math. Comput. Simul. 149, 69-90 (2018)

38. Xu, C.J., Liao, M.X., Li, P.L., Liu, Z.X., Yuan, S.: New results on pseudo almost periodic solutions of quaternion-valued fuzzy cellular neural networks with delays. Fuzzy Sets Syst. (2020). https://doi.org/10.1016/j.fss.2020.03.016

39. Xu, C.J., Li, P.L., Liao, M.X., Yuan, S.: Bifurcation analysis for a fractional-order chemotherapy model with two different delays. Math. Methods Appl. Sci. 43, 1053-1083 (2020)

40. Xu, C.J., Liao, M.X., Li, P.L., Guo, Y., Xiao, Q.M., Yuan, S.: Influence of multiple time delays on bifurcation of fractional-order neural networks. Appl. Math. Comput. 361, 565-582 (2019)

41. Hassard, B.D., Kazarinoff, N.D., Wan, Y.H.: Theory and Applications of Hopf Bifurcation. Cambridge University Press, Cambridge (1981)

42. Jana, D., Agrawal, R., Upadhyay, R.K.: Top-predator interference and gestation delay as determinants of the dynamics of a realistic model food chain. Chaos Solitons Fractals 69, 50-63 (2014)

43. Hu, G.P., Li, X.L.: Stability and Hopf bifurcation for a delayed predator-prey model with disease in the prey. Chaos Solitons Fractals 45, 229-237 (2012)

44. Xu, C.J., Liao, M.X., Li, P.L., Guo, Y.: Bifurcation analysis for simplified five-neuron bidirectional associative memory neural networks with four delays. Neural Process. Lett. 50, 2219-2245 (2019)

45. Xu, C.J., Liao, M.X., Li, P.L.: Bifurcation control of a fractional-order delayed competition and cooperation model of two enterprises. Sci. China, Technol. Sci. 62, 2130-2143 (2019)

46. Li, W., Huang, D.M., Zhang, M.T.: Bifurcation control of a generalized VDP system driven by color-noise excitation via FOPID controller. Chaos Solitons Fractals 121, 30-38 (2019) 